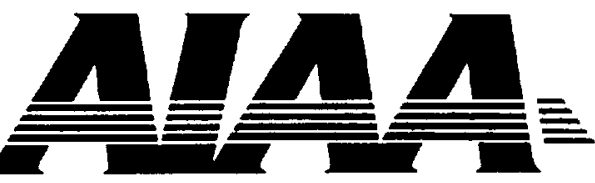

AIAA 96-2217

Calibration Improvements To ElectronicallyScanned Pressure Systems and Preliminary Statistical Assessment

Joel L. Everhart

NASA Langley Research Center Hampton, VA

19th AIAA Advanced Measure ment and G round Testing Technology Confere nce June 17-20, 1996/New Orleans, LA

For permission to copy or republish, contact the American Institute of Aeronautics and Astronautics 370 L'Enfant Promenade, S.W., Washington, D.C. 20024 



\title{
CALIBRATION IMPROVEMENTS TO ELECTRONICALLY-SCANNED PRESSURE SYSTEMS AND PRELIMINARY STATISTICAL ASSESSMENT
}

\author{
Joel L. Everhart ${ }^{\star}$ \\ NASA Langley Research Center \\ Hampton, VA 23681-0001
}

\begin{abstract}
Orifice-to-orifice inconsistencies in data acquired with an electronically-scanned pressure system at the beginning of a wind tunnel experiment forced modifications to the standard, instrument calibration procedures. These modifications included a large increase in the number of calibration points which would allow a critical examination of the calibration curve-fit process, and a subsequent post-test reduction of the pressure data. Evaluation of these data has resulted in an improved functional representation of the pressure-voltage signature for electronically-scanned pressures sensors, which can reduce the errors due to calibration curve fit to under 0.10 percent of reading compared to the manufacturer specified 0.10 percent of full scale. Application of the improved calibration function allows a more rational selection of the calibration set-point pressures. These pressures should be adjusted to achieve a voltage output which matches the physical shape of the pressure-voltage signature of the sensor. This process is conducted in lieu of the more traditional approach where a calibration pressure is specified and the resulting sensor voltage is recorded. The fifteen calibrations acquired over the two-week duration of the wind tunnel test were further used to perform a preliminary, statistical assessment of the variation in the calibration process. The results allowed the estimation of the bias uncertainty for a single instrument calibration; and, they form the precursor for more extensive and more controlled studies in the laboratory.
\end{abstract}

\section{Introduction}

Electronically-scanned pressure systems have become a mainstay of experimental

\footnotetext{
*Research Engineer, Senior Member AIAA

Copyright () 1996 by the American Institute of Aeronautics and Astronautics, Inc. No Copyright is asserted in the United States under Title 17, U.S. Code. The U.S. Government has a royaltyfree license to exercise all rights under the copyright claimed herein for govemment purposes. All other rights are reserved by the copyright owner.
}

testing because they provide a low-cost-perchannel instrumentation solution where large numbers of pressure measurements are required. These systems are computer controlled; they are relatively easy to use; they provide ondemand, "rapid" calibration techniques; and, they are capable of high accuracy when used in controlled, laboratory environments. However, as with any instrument, their use is pushed into originally unintended or unevaluated areas where measurement uncertainties may become large. Such an area was explored when pressures were acquired on the external nozzle expansion ramp of a powered, generic, hypersonic configuration which was tested (Everhart, Ashby, and Monta 1992, 1993) in the 20-Inch Mach 6 Tunnel at the NASA Langley Research Center (Miller and Smith 1986, Miller 1990). During this experiment an electronically-scanned pressure system was used to acquire pressures at 257 orifice locations on the model. The pressures measured on the nozzle expansion ramp, specifically, varied from near atmospheric values at the engine exhaust plane to about 0.01 atmospheres near the model trailing edge; in other words, accurate measurements were required over two orders of magnitude in pressure, and the instrumentation had to be selected to accommodate this variation. Several typical nozzle centerline distributions of local static pressure, $p$, normalized by the engine exit-plane pressure, $p_{4}$, are shown in Figure 1. Additionally, because the maximum freestream static pressure is on the order of 0.3 psi, the closest, logical reference pressure for the instrumentation is vacuum, which means that differential pressure gages must be used in the absolute sense by evacuating the reference side of the gage. Because the engine exhaustplane pressure and tunnel test conditions were varied widely, pressures on the nozzle at a fixed model station also varied widely; in many cases these pressures were outside the central portion of the sensor range, nominally 15 - to 85 -percent of full scale, where the most accurate measurements are typically achieved. In some cases the test conditions were such that the pressure at given orifice might drop below the 15-percent "limit" and exceed the 85-percent 
"limit" during the same wind tunnel run. The new pressure system was also required to measure nozzle pressures on the unpowered model where values near freestream were realized on all orifices simultaneously; thus, large range sensors were required to resolve small pressures. It is important to note that it was not feasible to modify the pressure orifice hookup between wind tunnel runs to more appropriately match measurement requirements with sensor capabilities because of the complexity of the model buildup and time restrictions imposed by the facility test schedule.

As a routine part of the initial setup and system checkout, the wind tunnel was evacuated to low pressure and data were recorded in a "wind-off" mode on all model pressure orifices. Significant, inconsistent, orifice-to-orifice pressure variations which could not be resolved by "tuning" the measurement system were revealed in these data. This tuning included among other things changing the calibration reference-pressure set points which modified the calibration coefficients, and changing the reference pressure dwell time to ensure pressure settling. Because the observed inconsistencies could not be removed, the calibration procedures were modified to allow greater flexibility in the post-test evaluation of the data and the pressure system calibration process. Even though these data were acquired during a hypersonic experiment, they are applicable to all situations where low pressure measurements with electronically-scanned pressure systems are used because of a similar response of the sensor near zero differential pressure. objectives:

This paper, then, has the following three

(1) It highlights some problems which exist in the currently used, standard calibration techniques.

(2) It presents a new functional representation of the characteristic pressure-voltage signature which markedly improves the measurement accuracy across the pressure range for electronically-scanned pressure gages. Additionally, this new functional representation leads to a more rational criteria for selecting the calibration pressures.

(3) This paper presents a preliminary statistical assessment of these calibration results. The assessment allows an estimate of the uncertainty in this calibration process, and it serves as a precursor to more controlled and detailed experiments with these instruments.

\section{Nomenclature}

$a_{i}, b, c$ General calibration function fitting coefficients, see eqn 1.

$A_{1}, A_{2}, A_{3}, A_{4}$ Calibration function fitting coefficients, see eqn 3 .

Bx Bias error.

$f \quad$ Linear sum of $g$ functions, see eqn. 7 .

g Arbitrary function, see eqn 7.

$G \quad$ Matrix of $g$ functions, see eqn 11.

$m, n$ Number of summation coefficients, see eqn 1.

Px Precision error.

p Pressure, psi

$p_{4} \quad$ Nozzle exit pressure, psi

Q Weighting function, see eqn 10.

$t \quad$ Student's t distribution

$U \quad$ Uncertainty

$\checkmark \quad$ Sensor output voltage, volt

$V_{0} \quad$ Zero-pressure offset voltage, volt

$X \quad$ Arbitrary independent variable.

$\bar{X} \quad$ Average of $X$ over $n$ repeats.

$Y \quad$ Measured value at $X$.

$\hat{Y} \quad$ Mathematical approximation to $Y$, see eqn 7.

$Z \quad$ Offset-referenced voltage, volt, see eqn 2

Subscripts

ref Reference

Abbreviations

Cl Confidence interval

ESP Electronically scanned pressure

F.S. Full scale

MLS Method of least squares

PI Prediction interval

Pnt Point

PRE Percent residual error, $|\Delta p| / p \times 100 \%$

Rdg Reading

RE Residual error, $\Delta p$, psi

SD Standard deviation, psi

SE Standard error, psi

\section{Experimental Procedure}

Specific information concerning the wind tunnel, model and experiment are discussed by Everhart, Ashby, and Monta (1992, 1993). This section presents a discussion of the instrumentation used to acquire the pressure data with particular emphasis on procedures used during the calibration process. 


\section{Instrumentation}

The System 8400 data acquisition instrumentation manufactured by Pressure Systems, Incorporated (PSI) was used to acquire the pressure data. The system may be controlled either from the front panel or by an external computer. Seven different electronically-scanned pressure (ESP) modules as enumerated in Table 1 were used simultaneously to measure the pressures. Also listed are the number of ports (sensors) per module and the manufacturer-specified uncertainty as a percent of full-scale pressure range.

If the ESP modules are maintained in a temperature-controlled laboratory environment, these systems are capable of high-accuracy measurements. This high accuracy occurs because temperature sensitivity effects on the zero-pressure offset voltage for each sensor are minimized due to the on-demand calibration capability of the system. It is the error introduced by temperature variation of the ESP module which may yield first-order variation in the calibration voltages, and, as a result, the calibration coefficients. When performing a standard calibration, a mechanical, internal, sliding block in each ESP module is used to isolate all sensors from their individual pressure orifice tubes; external calibration pressures are then applied to all ESP sensors simultaneously by the Pressure Standard Unit which contains a high accuracy $(0.02 \%$ FS) reference pressure standard; and, the output is digitized by the Scanner Digitizer Unit using a 16-bit analog-todigital converter. Calibrations in the present experiments were conducted immediately prior to each wind tunnel run, and the additional precaution of insulating the ESP modules was taken to further reduce the impact of temperature variations during the acquisition of test data. Stable ESP module temperatures were monitored throughout the calibration and test process.

The standard ESP calibration typically consists of either 3 or 5 data points which are used, respectively, to determine either a parabolic or a quartic polynomial representation of the pressure-voltage signature. As a result, the calibration curve, first, passes through the calibration data, encapsulating any random measurement error in the calibration coefficients, which, then propagates any error into the test data. Additionally, this polynomial calibration curve may or may not model the actual pressure-voltage signature of each ESP module sensor, particularly for small pressures or for pressures exceeding the range of the calibration data where the fitting polynomial may exhibit a non-physical behavior; thus, extrapolation of calibration results is very tenuous and uncertain.

For the present study, the differential ESP modules were used as absolute gages by evacuating the reference side of the modules using a common vacuum source. Because of curve-fit inadequacies when using the standard calibration techniques and because of calibration uncertainties experienced at the beginning of the study, the standard calibration method was unacceptable, and a different calibration procedure was used. In this procedure the sensor isolation block was slid into the calibrate position and a needle valve combined with a vacuum source was used to manually and successively apply 15 to 22 scheduled, calibration pressures to each of the ESP modules simultaneously. (Note that positive pressures were applied by venting to atmosphere.) Each calibration reference pressure was dialed into a digital channel on the data acquisition computer where it was recorded along with the digitized ESP voltage outputs of the System 8400 for post-test reduction.

When not in use, the ESP calibration block was slid into the calibrate position. Vacuum pressure was then applied to both the calibration and reference side of sensor to unload it to prevent the formation of a hysteresis bias. An important note is that the ESP sensors are structurally capable of sustaining the atmospheric differential pressure which was realized at the highest calibration pressures; however, a saturation voltage level of approximately 5 volts is achieved whenever the pressure exceeds the rated range of the ESP module, and these data were discarded when encountered.

Fifteen calibrations were made over the two-week duration of the study immediately prior to each wind tunnel run, each requiring $15-20$ minutes to execute because of the modified calibration procedures.

\section{Calibration-Pressure Working Standard}

The value of the calibration reference pressure was set using a Mensor DPG II (Digital 
Pressure Gauge, Model 14000) reference standard with a full scale range of $50 \mathrm{psi}$. The instrument has a compensated temperature range of $15^{\circ}$ to $35^{\circ}$ Centigrade. According to the UNCERTAINTY section in the Mensor operating guide (Mensor 1992)

"Measurement error due to the
combined effects of hysteresis,
repeatability, linearity and temperature
is $0.01 \%$ of full scale or less over the
compensated temperature range."

This yields an uncertainty of 0.005 psi which for the present test conditions represents $1.72 \%$ of freestream static pressure, $0.067 \%$ of freestream dynamic pressure, and $1.39 \%$ of the full-scale value of the 0.36-psi ESP module (i.e. the smallest module). While the Mensor range was larger than desired, it was the only pressure standard available on short notice and these uncertainties were deemed acceptable for the present application.

When selected the 50-psi Mensor was an acceptable choice. However, upon additional, post-test reading, the operating manual further states in the WARMUP section that after 30minute warm-up the zero drift of the instrument is $0.01 \%$ full scale per hour. Span drift is quoted as $0.005 \%$ full scale per week. Typical operating procedures are to leave the instrumentation in a power-on state and to zero the instrumentation at the start of a shift; thus, significant zero-drift error may occur prior to acquiring calibration data, particularly if problems which delay testing occur. This subject will be further addressed in the "Preliminary Statistical Assessment" section in this paper.

\section{Standard ESP Calibration Methods}

The most crucial portion of any measurement process is the calibration of the instrumentation. Generally, the quality of the calibration is only as good as the functional representation of the physical process being modeled; in the case of the ESP, this physical process is represented by the pressure-voltage signature of the sensor. Typical calibration data are shown in Figure $2 a$ for three ports, each from different ESP modules. The pressure data, $p$, are plotted on a linear scale for variations in output voltage, $V$. The port identification nomenclature is a single digit for the module combined with two digits for the port; for example, port 624 represents module 6 , port 24. Also shown in the inset is an expansion of the pressure region from 0 psi to 2 psi. Several observations can be made directly for all modules: first, away from zero pressure, the pressure-voltage signature is extremely linear; second, there appears to be significant nonlinearity near zero pressure; and, finally, there is a large variation in the zero-pressure offset value of the sensor.

The low-pressure variations are more readily apparent in Figure $2 b$ which replots the pressure logarithmically. Additional data have been added to those of Figure 2a to highlight the variations in zero-pressure offset within a single module; filled symbols represent the minimum value, while open symbols represent the maximum value. Offset results from the other ports on a given module are randomly dispersed between the limits presented.

The remainder of this section evaluates the standard, polynomial calibration methods used in ESP system hardware. Then, it then extends this by applying the method of least squares (MLS) to all of the calibration data to evaluate possible improvements in the calibration fitting. Finally, it presents an improved functional representation of the pressure-voltage signature whose coefficients are determined by least squares fitting; recommendations for implementation are given for the improved curve-fit formulation.

\section{Polynomial-Fitting Method}

The standard, supplied method of representing an ESP pressure-voltage signature is to fit an $n-1$ order curve through $n$ data points. Typically, the pressure data points are selected to cover the minimum and maximum expected values, and, then, equally spaced intervals are created within this range. Figure 3 considers calibration data from port 16 from each module, and examines how well successively higher order polynomials fit the unused, intermediate calibration data. Each of the figures are standardized to plot the calibration pressures ( $p$ ) and curve fit, the residual error $(\Delta p)$, and the magnitude of the percent of reading error $(|\Delta p| / p)$ versus output voltage. Figure $3 a$ presents the linear fit using just the two end points. In this case, using the data extremes will bias the calibration curve such that it consistently underestimates intermediate pressures. Residual errors (RE) in the lowpressure region peak at values near $0.15 \mathrm{psi}$ 
while peak percent-reading errors (PRE) lie in the 30-50 percent range for all modules. Additionally, any extrapolation of the calibration results toward zero pressure would be erroneous because the linear curve doesn't properly capture the roll off. A higher-order, parabolic representation is obtained by using a 3-point approximation and the results are shown in Figure 3b. Even though the data and the curve fit are in better agreement, the 2.5-psi module (for example) has a significant discrepancy of 0.05 psi at 1.5 volts (corresponding to 0.7 psi). This discrepancy exceeds the 0.0025 psi full-scale accuracy of the 2.5-psi module at 30 percent of the module range. Figure $3 \mathrm{c}$ presents a cubic fit of the data (4 points) which, again, offers little improvement to the calibration, and, in fact, shows a definite non-physical curvature within the range of the 0.36-psi data. Peak PRE values remain in the 8-45 percent range, and PRE is decreased for the 0.36 -psi modules only because the calibration curve is fitting error due to smaller numbers of available calibration pressures. The "best-fit" capability of the System 8400 is with five points which yields a quartic polynomial as shown in Figure 3d. Errors in the high pressure regions have been significantly reduced due to fitting more data points; however, unacceptably large errors (40-percent PRE) still exist in the low-pressure regions.

Figure 4 presents calibration-curve differences resulting from pressure set-point selection. The curves are standard 5-point (quartic) fits for port 16 on a 2.5-psi and port 16 on a 0.36-psi module. The open symbols represent all calibration data while the filled symbols are the data used to fit the curve. In the top case, large differences are visible between the calibration curve and the calibration points at the "error station" immediately to the left of the position labeled "Cal Set Point". In the bottom case, the "Cal Set Point" station has been interchanged with the "error station" and error is re-distributed to the right of the of "Cal Set Point". Additionally, the curves beyond the calibration range show very different, non-physical behavior. In other words, for the standard method, a judicious placement of the calibration data points is required to minimize the curve fitting error, as is an appropriate selection of the order of the fitting polynomial. It is important to note that the pressure-voltage signature for each sensor is unique and the best selection of calibration pressures will, therefore, also be unique and possibly vary from port to port.
Polynomial Fit Using Method of Least Squares

The method of least squares (MLS) was applied to the entire calibration data set (1) to minimize the "engineering-judgment" effects of calibration data point selection and (2) to obtain a rational definition of the fitting coefficients which allows for random error in the measurement process. The results using the calibration data of Figure 3 are shown in Figure 5 for linear (Fig. 5a), parabolic (Fig.5b), cubic (Fig.5c), and quartic (Fig.5d) MLS curve fits. Each of the figures are semi-logarithmic plots of the calibration pressures $(p)$ and curve fit, the residual error $(\Delta p)$, and the magnitude of the percent of reading error $(|\Delta p| / p)$ versus output voltage. Using this method, low-pressure PRE is reduced to about 10-percent compared to 30 50 percent with the Standard Method, while PRE of 0.1-1.0 percent are obtained in the high-pressure range. Because the pressurevoltage signature is represented by polynomials, the calibration curve will begin fitting error in the data, driving it to zero with successively higher-order terms, instead of fitting the actual functional representation of the data. Additionally, higher order polynomials may exhibit large oscillations within the range of the data even using MLS; this is known as the Runge Phenomena (ref. Young and Gregory, 1972).

\section{Improved ESP Functional Representation}

It was necessary to develop an alternate functional representation of the ESP pressurevoltage signature because of the previously cited data fitting inadequacies. A more general calibration function (compared to a standard polynomial) with the desired mathematical and physical properties is given by

$$
p=\sum_{i=2}^{m} a_{i} Z^{\frac{1}{i}}+b Z+\sum_{j=2}^{n} c_{j} Z^{j}
$$

where

$$
Z=V-V_{0}
$$

The $V_{0}$ is the sensor voltage at zero pressure and $a_{i}, b$, and $c_{j}$ are fitting coefficients. General properties of this function include:

(1) As $Z$ becomes large, the coefficients $b$ and $c_{j}$ dominate and a near-linear 
relationship may be retained for small $n$ and,

(2) As $Z$ approaches zero, the coefficient $a_{i}$ dominates and the low-voltage nonlinearity in the pressure signature (see Fig. 2a) can be modeled.

Specific criteria for selecting the values of $m$ and $n$ are that the resulting equation should be physically representative of the pressure-voltage signature, and the number of coefficients should be minimized to decrease data acquisition requirements. Because of previous experience and because the pressure data presented in figure $2 a$ show a distinctly linear trend as voltage becomes large, the value of $n$ was specified as 2 to capture any existing nonlinearity. Trial and error solutions showed it sufficient to specify $m$ as 3 . The resulting specific calibration function is written as

$$
p=A_{1} Z^{\frac{1}{3}}+A_{2} Z^{\frac{1}{2}}+A_{3} Z^{1}+A_{4} Z^{2}
$$

Because absolute zero pressure can never be obtained when the ESP modules are used as absolute gages, the true value of $V_{0}$ will never be realized in practice. Therefore, $V_{0}$ must be determined as part of the solution process, resulting in an "apparent" zero-pressure value.

The fitting function coefficients $A_{1}, A_{2}$, $A_{3}$, and $A_{4}$, and the zero-pressure voltage $V_{0}$ are iteratively determined in the following manner. First, the minimum voltage measured during the calibration is selected as the initial value of $V_{0}$ and used to compute a first estimate for $Z$ for each calibration pressure. The coefficients are determined using MLS (Press, et al. 1990) and used to evaluate the standard error of the calibration data about this candidate fitting curve. At this point, a new value of $v_{0}$ is selected and the process is repeated until minimum standard error convergence is obtained. (Note that $Z$ can never assume a negative value.) For ESP modules used as differential gages, the value of $V_{0}$ can be measured directly by unloading the sensor (i.e. venting both sides of the sensor to atmosphere), allowing $Z$ to be computed directly. Separate calibrations for positive and negative values of $Z$ may then be conducted, and positive-side and negative-side calibration coefficients may be iteratively obtained by using the absolute values of the calibration pressures and of $Z$.

Results of the fitting process applied to the improved calibration function are shown in
Figure 6 for port 16 (same data as that of Figures 2-5) on ESP modules with ranges of $0.36-, 2.5-, 5-$, and 15-psi. As in Figure 5, the pressure data and RE are plotted semilogarithmically versus $Z$ to highlight the differences at low pressure. The symbols represent the calibration data and the line represents the improved ESP fitting function. It is important to note that the RE and PRE scales have been reduced by an order of magnitude compared to previous results. Comparing the present results with Figure 5 (polynomial fitting with MLS) shows that maximum residual errors have been reduced from 0.04 to $0.006 \mathrm{psi}$. The maximum PRE values away from the immediate vicinity of the origin ( $Z=0$ volt) occur in the region corresponding to the knee ( $Z=0.5$ volt) of the pressure curve. Percent reading errors in this region have been reduced almost two orders of magnitude from about 10 percent for polynomial fitting with MLS to under 0.3 percent for the present method. Typical PRE values for the present method are 0.1 percent or less across the $Z$ range. Calibration curves from three different 0.36-psi and two different 15-psi ESP modules show that not only do similar calibrations exist within a module range but that consistent results are obtained across the range.

Typical coefficients for several curves shown in Figure 6 are given in Table 2 where a regular order in their values can be observed for increasing module range. Coefficients $A_{1}$ and $A_{2}$ monotonically increase and decrease, respectively, with module range, though the significance of these trends are at present unknown. The magnitudes of the slope coefficient, $A_{3}$ are typical and linearly increase with module range as with standard ESP calibration techniques. The linearity of the ESP sensors is further demonstrated by observing the small size of the nonlinear coefficient, $A_{4}$, as compared to the value of $A_{3}$. The size of $A_{4}$ is traditionally examined in the standard method to test for "calibration goodness". An additional indication of the goodness of the improved curve fit can be observed by examing the small size of the percent-full-scale error, defined as curve-fit standard error (SE) normalized by the full-scale module range, which is under $0.1 \%$. As previously demonstrated, this percent-full-scale error can be stated equally well as a percent-ofreading error.

Several properties of the present function can be observed by examining the equation, the results of Figure 6 , and the values 
in Table 2. First, in direct contrast to results presented for the other methods, this function properly captures the physical shape over all module ranges tested, especially in the lowpressure knee region about $Z=0.5$ volt. As a result, residual errors in pressure in both the absolute sense and as a percent of reading are very small compared to other methods across the entire pressure range of all modules tested. For large $Z$, the equation is a low-order, nearlylinear polynomial which allows a rational extrapolation toward the upper voltage limit of the sensor. For small $Z$, the equation reduces to a low-order root expansion which, again, allows a rational and consistent extrapolation toward the "apparent" zero-pressure value.

\section{Residual Error Assessment}

Residual errors (RE) are used to assess the calibration-method consistency for other sensor ports within a pressure range and when compared to other ranges. Residual errors for all ports obtained for a single calibration are presented in Figure 7 . The RE distributions are similarly biased in both magnitude and direction for each range which implies similarity of all calibrations (note that RE values are plotted for three 0.36-psi modules and two 15psi modules). Though not randomly distributed as ideally required by MLS theory, inclusion of higher-order calibration terms in the functional representation is unwarranted for the present case because immediately away from the origin RE are within the measurement accuracy of the calibration-pressure working standard.

\section{Effect of Calibration Data Distribution}

The sensitivity effects of the number of calibration pressures and their distribution on the calibration curve fit are plotted in Figure 8 for a 15-psi-range ESP module. The 22 calibration pressures are given by the open symbols and filled symbols are used to denote the evaluation subsets which include cases with $8,7,6$, and 5 pressures. Three 5-point cases were selected with various set-point arrangements because five points represent the minimum number for MLS fitting with four coefficients. Five points also helps minimize the time required to calibrate, which enhances wind tunnel productivity. Solid lines are used for the complete 22-point calibration, while dashed lines are used for the subset calibrations. The pressure-selection criteria for the subset evaluations was to anchor the ends of the range (i.e. the maximum and minimum values), select a pressure in the midrange, and to distribute the remaining pressures in the knee region. In each case, the dashed subset lines are indistinguishable from the solid completecalibration lines. Values of the standard error, the curve-fit coefficients, and apparent $V_{0}$ are presented in Table 3 for the cases plotted in Figure 8. Reducing the number of calibration pressures has little effect on the standard error until five points is reached. At this point, few degrees freedom exist so little variability is allowed; therefore, standard error is driven close to zero. The apparent zero-pressure voltage is virtually unaffected and varies only 0.00006 volts from minimum to maximum. Coefficients $A_{1}, A_{2}, A_{3}$, and $A_{4}$ have only a small variation with the largest variations appearing in $A_{1}$ and $A_{2}$ as expected. This insensitivity to number of points and location is a further indication that the physics (though empirically derived) are properly modeled with this mathematical representation.

\section{Recommended Calibration Technique}

The typical calibration philosophy is to prescribe a schedule of calibration pressures, record the resulting sensor voltage output, and curve fit these data with a standard polynomial. As has been demonstrated, this procedure can lead to substantial curve fit errors. It has also been demonstrated that the pressure-voltage relationship of the ESP module has a definite signature, that equation (3) is a significantly improved mathematical model of this signature, and that the curve fit is relatively insensitive to both number of points and their location. Therefore, based on current understanding, a new paradigm for calibrating ESP modules has emerged wherein the calibration pressure should be applied to generate a voltage output near specific values of $Z$. These positions include a minimum of 5 pressure settings which capture the significant features of the calibration curve, including the minimum ( $Z=0.0$ volts), below the knee ( $Z=0.25$ volts), above the knee $(Z=1.0$ volt), in the midrange $(Z=2.5$ volts), and near the maximum $(Z=4.5$ volts). Because of the insensitivity of the curve, $Z$ may be approximated by initially using as $V_{0}$ that value corresponding to near vacuum pressure (a final $Z$ will result from the curve-fit process). Obviously, additional pressures are desirable to reduce the curve fit uncertainty; however, they may not be required with improved pressure standards and data acquisition procedures, and with further experience. 


\section{Preliminary Statistical Assessment}

A preliminary statistical evaluation of the ESP calibration is presented in this section. The present data were acquired over a two-week period and include changes in the System 8400 instrumentation and model hookup, as well as temperature cycling due to the operation of the wind tunnel. The present data, while sufficient for a wind tunnel experiment, are insufficient to perform a detailed, statistical characterization of the ESP calibration process, because data which would be obtained under more controlled, laboratory conditions were not acquired. Additional data required to satisfy these more stringent conditions would include, for instance,

(1) extensive back-to-back calibrations to assess error from random effects,

(2) precise control of the sensor temperature to evaluate zero-pressure voltage variations,

(3) more precise control and recording of calibration set-point pressure,

(4) smaller range pressure standards,

(5) variations in ambient pressure, and

(6) vibration effects.

What the present data do allow is a statistical evaluation of the functional representation of the pressure-voltage signature, a near-term (over a 2-week period) statistical look at calibration coefficient variability in a real-world environment, and an estimate of systematic errors in the calibration. The first subsection presents some fundamental statistical concepts. In depth discussions of the required statistics can be obtained in standard texts such as Coleman and Steele (1989).

\section{Fundamental Statistical Concepts}

Generally, a single data value, $X$, is the best estimate, $X_{\text {measured }}$ of the true value to within an uncertainty, $U_{x}$, at a specified level of confidence. That is,

$$
X=X_{\text {measured }} \pm U_{X}
$$

where the uncertainty may be further separated into precision error, $P_{X}$, and bias error, $B_{x}$. The uncertainty is given by

$$
U_{X}^{2}=B_{X}^{2}+P_{X}^{2}
$$

Bias errors are the systematic offset of a measurement from its true value. Bias errors are often erroneously excluded when quoting measurement accuracies; many times they are excluded because they must be referenced to some recognized absolute which is difficult to obtain. Precision errors are random measurement errors. Typically, precision errors have a Gaussian or normal distribution about the mean value. A measure of the spread of this distribution about the mean is given by the standard deviation. Standard deviation is defined as

$$
\sigma_{X}=\sqrt{\sum_{i=1}^{n} \frac{\left(X_{i}-\bar{X}\right)^{2}}{(n-1)}}
$$

where $\bar{X}$ is the mean value of the $X_{i}$ taken over $n$ repeats.

For a series of measured data values, $Y$, at independent variable $X$, the approximating functional variation, $\hat{Y}$, through these values is given by

$$
\begin{aligned}
\hat{Y}(X) & =a_{1} g_{1}(X)+a_{2} g_{2}(X)+\ldots+a_{k} g_{k}(X) \\
& =f\left(X ; a_{j}\right), \quad \text { with } \quad j=1,2, \ldots, k
\end{aligned}
$$

where $f$ is the linear combination of the functions $g_{j}$. The coefficients $a_{j}$ are, typically, determined in a "best-fit" sense using techniques such as the Method of Least Squares (Brownlee 1965) which minimize the data scatter about the approximating function $\hat{Y}(X)$, usually by assuming a normal or Gaussian distribution. A polynomial is an example of a function $f$ where the $g_{1}, g_{2}, g_{3}, \ldots$ functions would be given by 1 , $x_{1} x^{2}, \ldots$, respectively.

Many measures of "curve-fit goodness" are available. One is given by the standard error defined as

$$
S E=\sqrt{\sum_{i=1}^{n} \frac{\left[Y_{i}-\hat{Y}\left(X_{i}\right)\right]^{2}}{(n-k)}}
$$

where $Y_{i}$ is the measured data value and $\hat{Y}$ is the approximated value at $X_{j}$. The number of degrees of freedom is given by the difference between the number of data values, $n$, and the number of coefficients, $k$. The number of degrees of freedom is important for flexibility in mathematically "fairing" the curve through the 
data trend, in contrast to "fitting" the curve through the data value. Another measure of goodness is given by the confidence interval, $\mathrm{Cl}$, which defines an uncertainty band at a specified level of confidence about the curve $\hat{Y}$ and within which the true value is predicted to lie. Following the nomenclature of Wahls, et al. (1995), confidence interval at the point $X_{0}$ is defined as

$$
C I\left(X_{0}\right)= \pm t_{\alpha / 2, v} \times S E \times Q\left(X_{0}\right)
$$

where $t_{\alpha / 2, v}$ is the value of the student's $t$ distribution at the $\alpha$ confidence level for $v=n-k$ degrees of freedom. The term $Q\left(X_{0}\right)$ is a weighting function based on data density and is defined as

$$
Q\left(X_{0}\right)=\sqrt{G_{0}^{T}\left(G^{T} G\right)^{-1} G_{0}}
$$

where

$$
G=\left[g_{1}, g_{2}, g_{3} \ldots g_{k}\right]_{k x 1}
$$

is a matrix of the $g_{j}$ functions of equation (7), and the transpose of $G$ evaluated at $X_{0}$ is given by

$$
G_{0}^{T}=\left[g_{1}\left(X_{0}\right), g_{2},\left(X_{0}\right), g_{3}\left(X_{0}\right), \ldots, g_{k}\left(X_{0}\right)\right]_{1 \times k}
$$

A final, necessary, statistical concept is that of the prediction interval, $P$. A prediction interval is the uncertainty band about the curve $\hat{Y}$ within which a future value or measurement is projected to lie based on available (historical) results and assuming the measurement process is unchanged. Prediction interval is defined as

$$
P I= \pm t_{\alpha / 2, v} \times S E \times \sqrt{1+Q\left(X_{0}\right)^{2}}
$$

It is important to note that through $Q$ the $C l$ and $P$ l are functions of the data density; therefore, they will generally have a narrower band in those regions where data density is the greatest since curve fits are generally more accurate there. The band will expand near the extremes where fewer data points usually exist. For the present paper, all uncertainties are taken at the $95 \%$ confidence level.

\section{Coefficient Averages and Standard Deviations}

The coefficient averages and standard deviations for each module for a single calibration are presented in Table 4 . For coefficients $A_{1}, A_{2}$, and $A_{3}$ the averages are well behaved in that they exhibit a linear variation with module range and the standard deviations are, typically, an order of magnitude smaller than the coefficient averages, except for the smallest range where less data are available at the lowest pressures for fitting. The standard deviation of the $A_{4}$ coefficients is of comparable size with the average coefficient; this is an additional indication of the linearity of the pressure-voltage signature for large $Z$. A visual examination of the curve-fit plots and coefficient tabulations, the consistency of the coefficient averages and standard deviations, and the small size of the residual errors validates the empirical, mathematical modeling of the physical process.

\section{Calibration Curve and Coefficient Variability}

An example of the calibration-tocalibration variability is given in Figure 9 for the 15 separate ESP calibrations for port 16 from the 2.5-psi module. Fourteen calibrations for this port are contained within a tight band. These fourteen calibrations were obtained for a stable hardware configuration. The outlying, initial curve was obtained prior to a System 8400 hardware modification and demonstrates an effect of changing the measurement process. This outlying curve also demonstrates the need for end-to-end system characterization, calibration, and configuration control. Generally, the fourteen calibration curves are similar; however, the expanded scale shows visible variation occurring among the curves in the knee region below $Z=1.0$ volt. Control charts representing the historical variation of the curve coefficients are given in Figure 10. Generally, the coefficients contain only small variation between successive cases with the most significant difference occurring for the initial case. The relative invariance of $V_{0}$ for the larger range modules (Fig. 10a) is indicative of the general stability of the environmental temperature. Increased $V_{0}$ variability is expected and is visible for the 0.36 psi modules because the calibration pressure points are fewer and more evenly distributed over the module range compared to the dense data distribution at low pressures for the higher range modules (see Figure 2).

Calibration curve variational trends for the different pressure modules were further examined by subtracting a reference calibration for each module from the other calibrations of 
the same module. The resulting incrementalpressure expression

$$
\begin{aligned}
p-p_{\text {ref }}= & \left(A_{1}-A_{1, \text { ref }}\right) Z^{\frac{1}{3}}+\left(A_{2}-A_{2, \text { ref }}\right) Z^{\frac{1}{2}} \\
& +\left(A_{3}-A_{3, \text { ref }}\right) Z^{1}+\left(A_{4}-A_{4, \text { ref }}\right) Z^{2}
\end{aligned}
$$

was evaluated at $Z=2$ volts using calibration 7 as the reference, and the results are plotted in Figure 11. This incremental representation collapses the calibrations and it displays an obvious wavy trend in the calibration history. Because all modules are affected similarly, the cause of the trend is independent of the module and must be the result some external influence. The exact reason for this variation is unknown; however, part of the shift is believed to be the result of a drifting zero setting on the calibration-pressure working standard. If this is indeed the case, the reference standard would have a potential full-scale root-mean-square error (including transducer uncertainty plus zero-drift uncertainty) of $\pm\left[(0.01)^{2}+(0.01)^{2}\right]^{1 / 2}= \pm 0.014 \%$ or \pm 0.0071 psi. This would be particularly significant at the smallest pressures where it would translate into uncertainties in the $A_{1}$ and $A_{2}$ coefficients, which would most affect the calibration-curve knee region.

\section{Estimate of Calibration Curve Uncertainty}

Any constant, unknown shift in the data for a single calibration caused by an external error source (ex. erroneous zero setting on the pressure standard) is a bias error which, if uncorrected, becomes encapsulated in the final data. If these bias errors randomly vary between calibrations, then they become part of the measurement imprecision for the entire test. This calibration imprecision can be analyzed by examining the variability of the aggregate calibration data set, which will yield a estimate of the bias uncertainty for a single calibration. Random variation has been demonstrated for the present calibration curves (see Figure 11), and sufficient data are available (fourteen process-stable calibrations) for statistical assessment of the bias as follows. First, the individual, previously-determined, apparent zero-pressure voltages are subtracted from each data set and the data are, then, grouped into a single aggregate. Finally, the improved calibration curve (equation 3 ) is generated for this aggregate data set, and, $\mathrm{Cl}$ and $\mathrm{Pl}$ statistics (eqns. 9 and 13 , respectively) are determined. The results for modules in each pressure range are shown in Figure 12 where the symbols are the aggregate data for port 16 and the solid lines represent the aggregate curve fit. (Note, again, that the pressure scale is logarithmic.) The nominal magnitudes of the $\mathrm{Cl}$ and $\mathrm{Pl}$ at the $95 \%$ level of uncertainty are 0.003 and 0.020 psi. Normally, these values would be presented as positive and negative increments about the curve; however, because of their small size they are presented separately for clarity. Confidence interval increases somewhat at the higher calibration pressures because there are fewer data available to fit in this region. The individual curves are not distinguished according to module range because the differences are negligible for the present purpose. Recall that the $\mathrm{Cl}$ represents the uncertainty band about the estimated calibration curve within which the true curve will lie 95-percent of the time. The small $\mathrm{Cl}$ value statistically implies that the estimated calibration curve is very representative of the of the true curve for these aggregate data. Recall also that the $P I$ is based on historical data and that it represents the uncertainty band about the estimated curve within which a future calibration curve would lie assuming that no changes in the calibration process occur. The bias uncertainty is 0.04 -percent of the full-scale range of the calibration standard which exceeds the 0.01-percent accuracy specification of the instrument. By taking the root-mean-square difference between the potential error sources (0.01-percent accuracy specification and a 0.01 -percent zero shift) and the actual 0.04percent measurement, an additional bias uncertainty of 0.014 percent is obtained for all other, unknown, sources.

\section{Estimate of Pressure Uncertainty}

Uncertainties due to temperature variations are considered negligible because:

(1) the ESP modules were calibrated prior to each run which removes the first order effect of module temperature variation,

(2) the ESP modules are mounted in the injection chamber beneath the wind tunnel, which is isolated from the high-temperature stream by a solid floor plate,

(3) the ESP modules were insulated and stable temperatures were recorded on the external surface of the module case during each run, and

(4) the relative invariance of the $V_{0}$ presented in Figure 10a, particularly for the larger 
range modules, does not imply any significant temperatures excursions.

Back-to-back repeat calibrations required to assess uncertainties due to shortterm randomness in the pressure were not acquired. However, as per Table 1, the manufacturer specifications for these instruments are $0.1 \% \mathrm{FS}$ for modules in the range of 0.36 psi to $2.5 \mathrm{psi}$ and $0.05 \% \mathrm{FS}$ for modules in the range of 5 psi to $15 \mathrm{psi}$.

Uncertainties due to calibration curve fit have been identified herein to be $0.1 \%$ of reading or less.

An estimate of the bias error from all external sources has been shown to be four times the manufacturer specified level of $0.01 \%$ FS for the calibration standard. This error is a constant \pm 0.02 psi for all ESP modules. The most likely error source is an erroneous zero setting on the reference standard.

For pressure data acquired using the present ESP calibration data, these estimates yield bias and precision uncertainties of

$$
B_{P}{ }^{2}=(0.02)^{2}+(0.001 x p)^{2}
$$

and,

$$
\begin{aligned}
P_{P}^{2} & =\left(0.0005 x p_{F S}\right)^{2}, p_{F S} \geq 5 \mathrm{psi} \\
& =\left(0.0010 x p_{F S}\right)^{2}, p_{F S}<5 \mathrm{psi}
\end{aligned}
$$

where $p_{F S}$ is the full-scale range of the ESP module under consideration.

\section{Concluding Remarks}

Pressure measurement inconsistencies which occurred during the conduct of a wind tunnel experiment prompted a critical examination of the calibration process and the mathematical relationship used to model the pressure-voltage signature of an electronicallyscanned pressure system. This examination revealed deficiencies and inadequacies with the current calibration techniques. Specifically, the standard 3-point parabolic-fit and 5-point quartic-fit calibration methods are insufficient in that (1) they do not capture the low-pressure variation of the sensor, and (2) that any extrapolation beyond the calibration data range to either low or high pressures is tenuous due to the Runge Phenomena, which is a mathematical oscillation inherent in higherorder polynomials. Furthermore, extension of the standard polynomial fitting method by the method of least squares offers little improvement to the calibration because of (1) inappropriate modeling of the pressure-voltage signature, (2) the uncertainty associated with the selection of the calibration set points, and (3) the possible deviations due to fitting-curve oscillations within the range of the data, again, due to the Runge Phenomena. A new functional representation of the pressure-voltage signature has been developed and demonstrated to offer significant calibration improvements for low to high pressures, and it is applicable over all tested ESP module sizes. Calibration coefficients resulting from this procedure are well-behaved in that they have a regular variation and, where applicable, are comparable in magnitude to those obtained using standard techniques. In addition, the calibration curve varies properly within the data range and it can be safely extrapolated to the voltage limit of the sensor. The improved representation allows these instruments to achieve 0.1-percent of reading accuracy (or better) across the module pressure range.

With the improved calibration function, an alternate calibration procedure has emerged wherein calibration pressures should be adjusted to achieve a specific schedule of calibration voltages. This procedure is proposed in lieu of the more traditional approach where a calibration pressure is specified and the resulting voltage is recorded. In this revised approach, the calibration voltages are selected to match the physical characteristics of the well-defined pressurevoltage signature, resulting significantly reduced inaccuracy and consistent calibration coefficients.

With curve-fit inaccuracies for the individual calibrations reduced to a second order effect, valid comparisons among the calibrations could be made. These comparisons revealed random pressure variations occurring simultaneously on all ESP modules, indicating a common, external bias. The magnitude of the bias uncertainty was evaluated using data from fourteen calibration repeats acquired over a 2-week period. This bias uncertainty was found to be 0.020 psi, which is four times the quoted uncertainty of the calibration-pressure working standard $(0.005$ psi). 
The process just described is the first of several such cycles required to achieve statistical process control of electronicallyscanned pressure measurements and instrumentation. This preliminary calibration data base revealed several problems, corrective action was taken, and an assessment of the results was made. The next cycle is to reexamine this measurement process in the laboratory under more controlled conditions and to implement improved procedures and methods in the wind tunnel environment. The resulting product will be quality wind tunnel pressure data.

\section{Acknowledgments}

The author would like to thank $\mathrm{Dr}$. Michael J. Hemsch of Lockheed-Martin Engineering \& Sciences Co. (assigned to Aerodynamics Division, Langley Research Center) for serving as a sounding board and for many fruitful conversations concerning data quality.

\section{References}

Aeschliman, D. P.; Oberkampf, W. L.; and Henfling, J. F. 1994: Fast-Response, Electronically-Scanned Multi-Port Pressure System for Low-Pressure Hypersonic Wind Tunnel Applications. AIAA 94-2580.

Brownlee, K. A. 1965: "Statistical Theory and Methodology In Science and Engineering", Second Edition, John Wiley \& Sons, New York.

Coleman, Hugh W.; and, Steele, W. Glenn 1989: "Experimentation and Uncertainty Analysis for Engineers", Interscience-John Wiley \& Sons, New York.

Everhart, Joel L.; Ashby, George C., Jr.; and, Monta, William J 1992.: "Test Description and
Preliminary Pitot-Pressure Surveys for Langley Test Technique Demonstrator at Mach 6", AIAA 92-3940.

Everhart, Joel L.; Ashby, George C., Jr.; and, Monta, William J. 1993: "Experimental Surface Pressure and Flow Field Results on an Powered Simulation Model at Mach 6", Paper Number 37. Presented at the National Aero-Space Plane Technology Review, Monterey, CA.

Operating Manual for DPGII Model 14000 Digital Pressure Gauge. Manual Part Number 00114024001D 150, Mensor Corporation, San Marcos, Texas, Aug. 1992.

Miller, C.G.; and, Smith, F.M. 1986: "Langley Hypersonic Facilities Complex - Description and Application", AlAA Paper No. 86-0741.

Miller, C.G. 1990: "Langley Hypersonic Aerodynamic/Aerothermodynamic Testing Capabilities - Present and Future", AIAA 901376.

Press, William H.; Flannery, Brian P.; Teukolsky, Saul A.; and Vetterling, William T. 1990: "Numerical Recipes: The Art of Scientific Computing (Fortran) ${ }^{n}$, Cambridge University Press, Cambridge.

Taylor, Barry N.; and, Kuyatt, Chris E. 1994: "Guidelines for Evaluating and Expressing the Uncertainty of NIST Measurement Results", NIST Technical Note 1297.

Wahls, R. A.; Adcock, J. B.; Witkowski, D. P.; and Wright, F. L.: "A Longitudinal Aerodynamic Data Repeatability Study for a Commercial Transport Model Test in the National Transonic Facility", NASA Technical Paper 3522.

Young, David M.; and Gregory, Robert Todd 1972: "A Survey of Numerical Mathematics", Vol. 1, Addison-Wesley Publishing Company, Inc., Reading, Massachusetts 


\section{TABLES}

\begin{tabular}{|c|c|c|c|}
\hline $\begin{array}{c}\text { Module } \\
\text { Number }\end{array}$ & $\begin{array}{c}\text { Range } \\
\text { (psi) }\end{array}$ & $\begin{array}{c}\text { Number of } \\
\text { Ports }\end{array}$ & $\begin{array}{c}\text { Full-Scale } \\
\text { Uncertainty }\end{array}$ \\
\hline 1 & 2.5 & 48 & $+1-0.1 \%$ \\
\hline 2 & 5.0 & 48 & $+/-0.05 \%$ \\
\hline 3 & 15.0 & 48 & $+1-0.05 \%$ \\
\hline 4 & 15.0 & 48 & $+/-0.05 \%$ \\
\hline 5 & 0.36 & 32 & $+1-0.1 \%$ \\
\hline 6 & 0.36 & 32 & $+1-0.1 \%$ \\
\hline 7 & 0.36 & 32 & $+1-0.1 \%$ \\
\hline Total & & 288 & \\
\hline
\end{tabular}

Table 1.- Electronically-scanned pressure modules used with manufacture-specified, full-scale uncertainty specifications.

\begin{tabular}{|c|c||c|c|c|c||c|c|}
\hline Module & $\begin{array}{c}\text { Range } \\
\text { (psi) }\end{array}$ & $\mathbf{A}_{\mathbf{1}}$ & $\mathbf{A}_{\mathbf{2}}$ & $\mathbf{A}_{\mathbf{3}}$ & $\mathbf{A}_{4}$ & SE & $\begin{array}{c}\text { SE/Range } \\
(\%)\end{array}$ \\
\hline 5 & 0.36 & 0.0437 & 0.1032 & 0.0445 & 0.0022 & 0.0003 & 0.095 \\
\hline 1 & 2.5 & 0.2241 & 0.0141 & 0.5334 & 0.0083 & 0.0017 & 0.069 \\
\hline 2 & 5 & 0.4092 & -0.1422 & 1.1388 & 0.0072 & 0.0035 & 0.070 \\
\hline 3 & 15 & 0.6676 & -0.5038 & 3.5211 & 0.0026 & 0.0035 & 0.024 \\
\hline
\end{tabular}

Table 2.- Typical fitting function coefficients for curves in Figure 6.

\begin{tabular}{|c|c|c|c|c|c|c|c|}
\hline $\begin{array}{c}\text { No. } \\
\text { Pts }\end{array}$ & $\begin{array}{c}\text { Pts } \\
\text { Used }\end{array}$ & $\begin{array}{c}\text { Std } \\
\text { Error }\end{array}$ & $\mathbf{V}_{\mathbf{0}}$ & $\mathbf{A}_{\mathbf{1}}$ & $\mathbf{A}_{\mathbf{2}}$ & $\mathbf{A}_{\mathbf{3}}$ & $\mathbf{A}_{\mathbf{4}}$ \\
\hline 22 & all & 0.00178 & .03596 & .12130 & -.11864 & 3.51447 & -.00114 \\
\hline 8 & $1,3,7,11,12,15,19,22$ & 0.00174 & .03600 & .13960 & -.14791 & 3.52571 & -.00195 \\
\hline 7 & $1,3,11,12,15,19,22$ & 0.00193 & .03601 & .14657 & -.15765 & 3.52859 & -.00214 \\
\hline 6 & $1,3,11,15,19,22$ & 0.00232 & .03601 & .14500 & -.15397 & 3.52654 & -.00193 \\
\hline 5 & $1,11,13,19,22$ & $4 \times 10^{-7}$ & .03595 & .08036 & -.05771 & 3.49379 & .00059 \\
\hline 5 & $1,11,14,19,22$ & $3 \times 10^{-7}$ & .03596 & .08138 & -.05933 & 3.49439 & .00055 \\
\hline 5 & $1,11,15,19,22$ & $1 \times 10^{-7}$ & .03599 & .11311 & -.11003 & 3.51313 & -.00092 \\
\hline
\end{tabular}

Table 3.- Effect of number of fitting points on calibration coefficients.

\begin{tabular}{|c|c||c|c|c|c||c|c|c|c|}
\hline $\begin{array}{c}\text { ESP } \\
\text { No. }\end{array}$ & $\begin{array}{c}\text { FS } \\
\text { (psi) }\end{array}$ & $\begin{array}{c}\text { Avg } \\
\mathbf{A}_{1}\end{array}$ & $\begin{array}{c}\text { Avg } \\
\mathbf{A}_{2}\end{array}$ & $\begin{array}{c}\text { Avg } \\
\mathbf{A}_{3}\end{array}$ & $\begin{array}{c}\text { Avg } \\
\mathbf{A}_{4}\end{array}$ & $\begin{array}{c}\text { SD } \\
\mathbf{A}_{1}\end{array}$ & $\begin{array}{c}\text { SD } \\
\mathbf{A}_{2}\end{array}$ & $\begin{array}{c}\text { SD } \\
\mathbf{A}_{3}\end{array}$ & $\begin{array}{c}\text { SD } \\
\mathbf{A}_{4}\end{array}$ \\
\hline 5 & 0.36 & 0.0482 & 0.0972 & 0.0464 & 0.0020 & 0.0236 & 0.0284 & 0.0082 & 0.0006 \\
\hline 6 & 0.36 & 0.0380 & 0.1106 & 0.0445 & 0.0024 & 0.0123 & 0.0159 & 0.0041 & 0.0004 \\
\hline 7 & 0.36 & 0.0586 & 0.0893 & 0.0513 & 0.0023 & 0.0285 & 0.0370 & 0.0102 & 0.0010 \\
\hline 1 & 2.5 & 0.2518 & -0.0277 & 0.5561 & 0.0060 & 0.0230 & 0.0333 & 0.0176 & 0.0016 \\
\hline 2 & 5 & 0.4115 & -0.1416 & 1.1378 & 0.0077 & 0.0178 & 0.0264 & 0.0213 & 0.0014 \\
\hline 3 & 15 & 0.6889 & -0.5285 & 3.4951 & 0.0034 & 0.0141 & 0.0172 & 0.0555 & 0.0011 \\
\hline 4 & 15 & 0.6545 & -0.5133 & 3.5129 & 0.0041 & 0.0125 & 0.0152 & 0.0686 & 0.0020 \\
\hline
\end{tabular}

Table 4.- Variation of calibration coefficients within a module. 


\section{Figures}

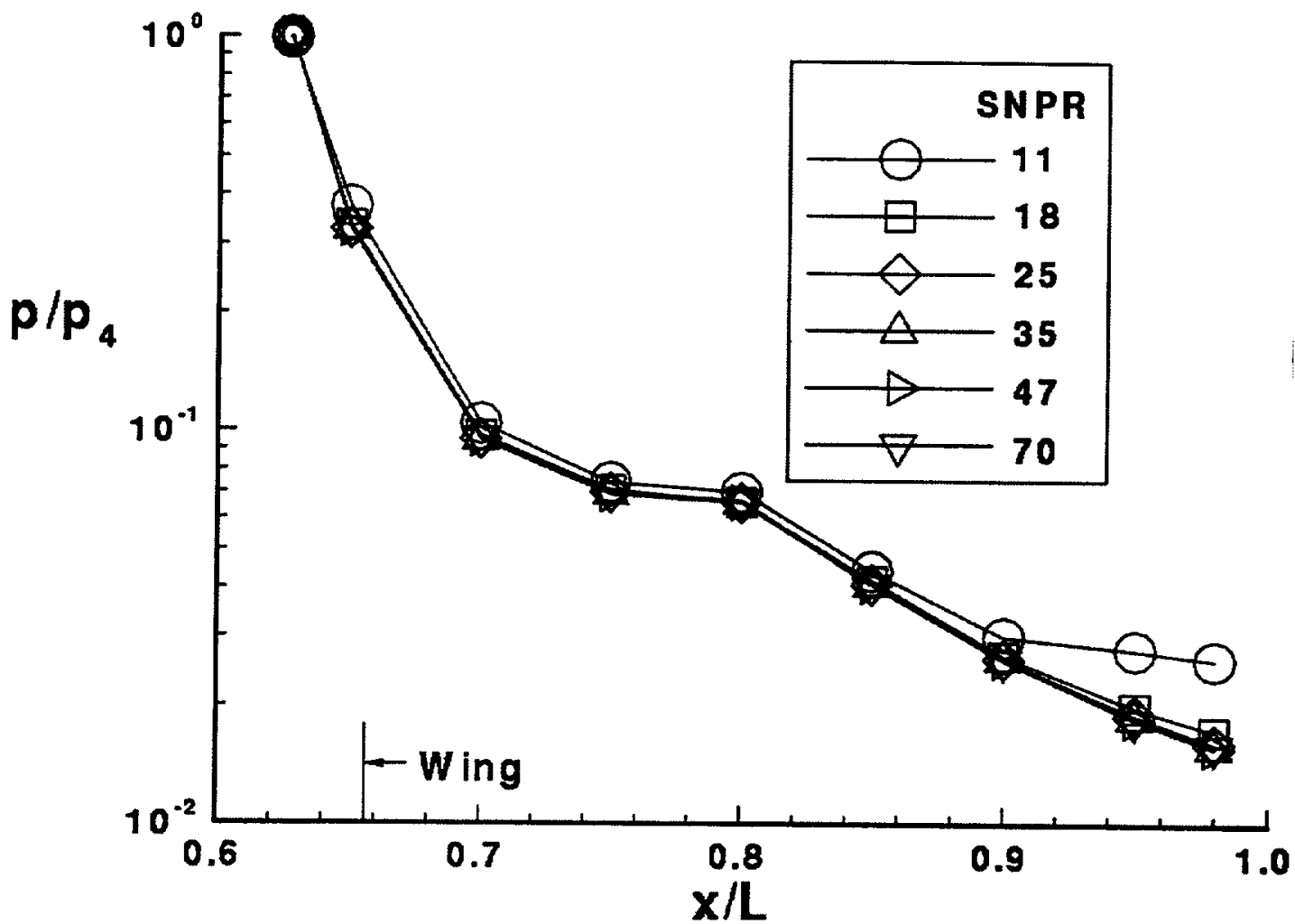

Figure 1.- Effect of static nozzle pressure ratio (SNPR) on external, centerline, nozzle pressure ratio on a generic hypersonic propulsion simulation model. 


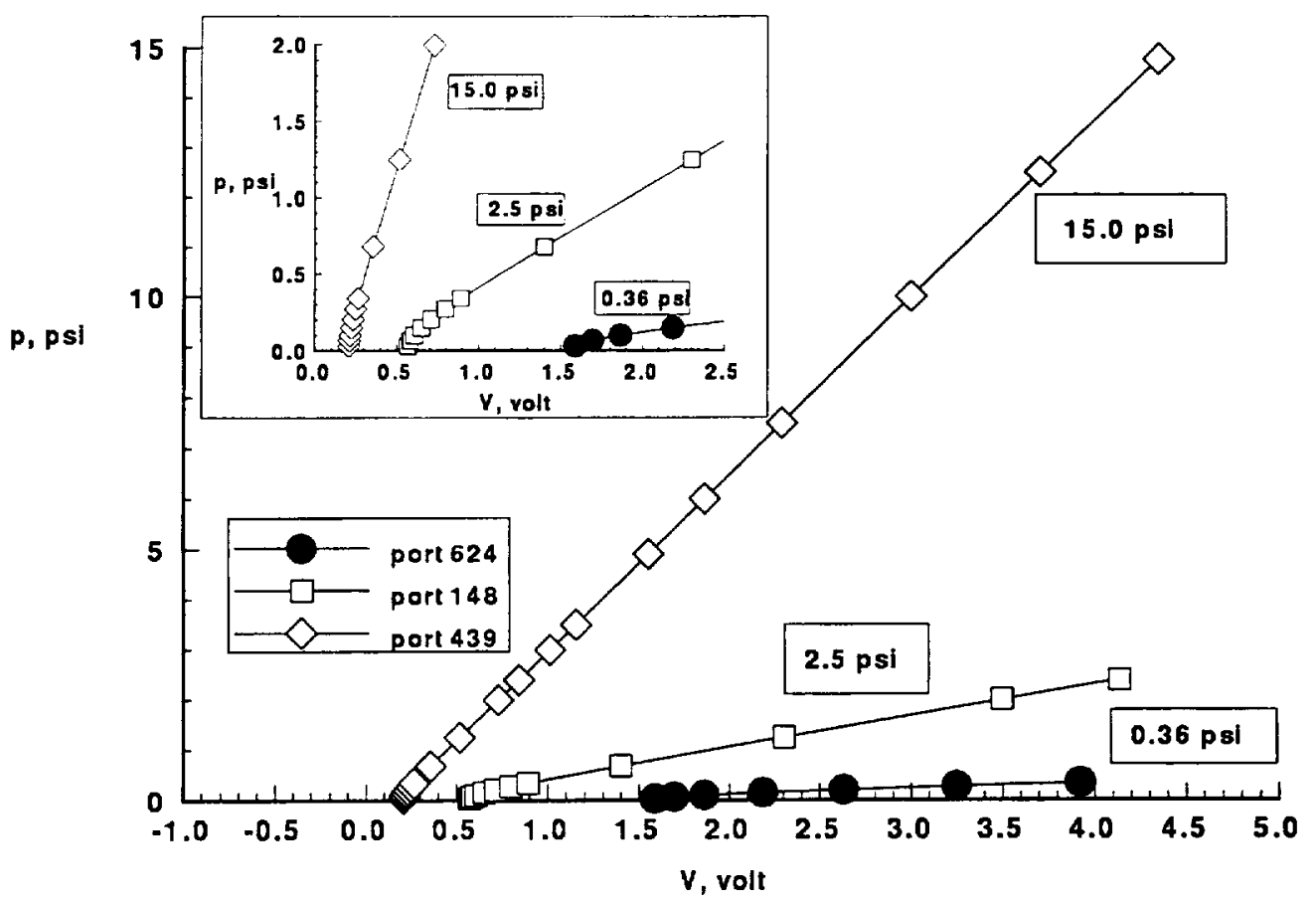

a. Linear scale.

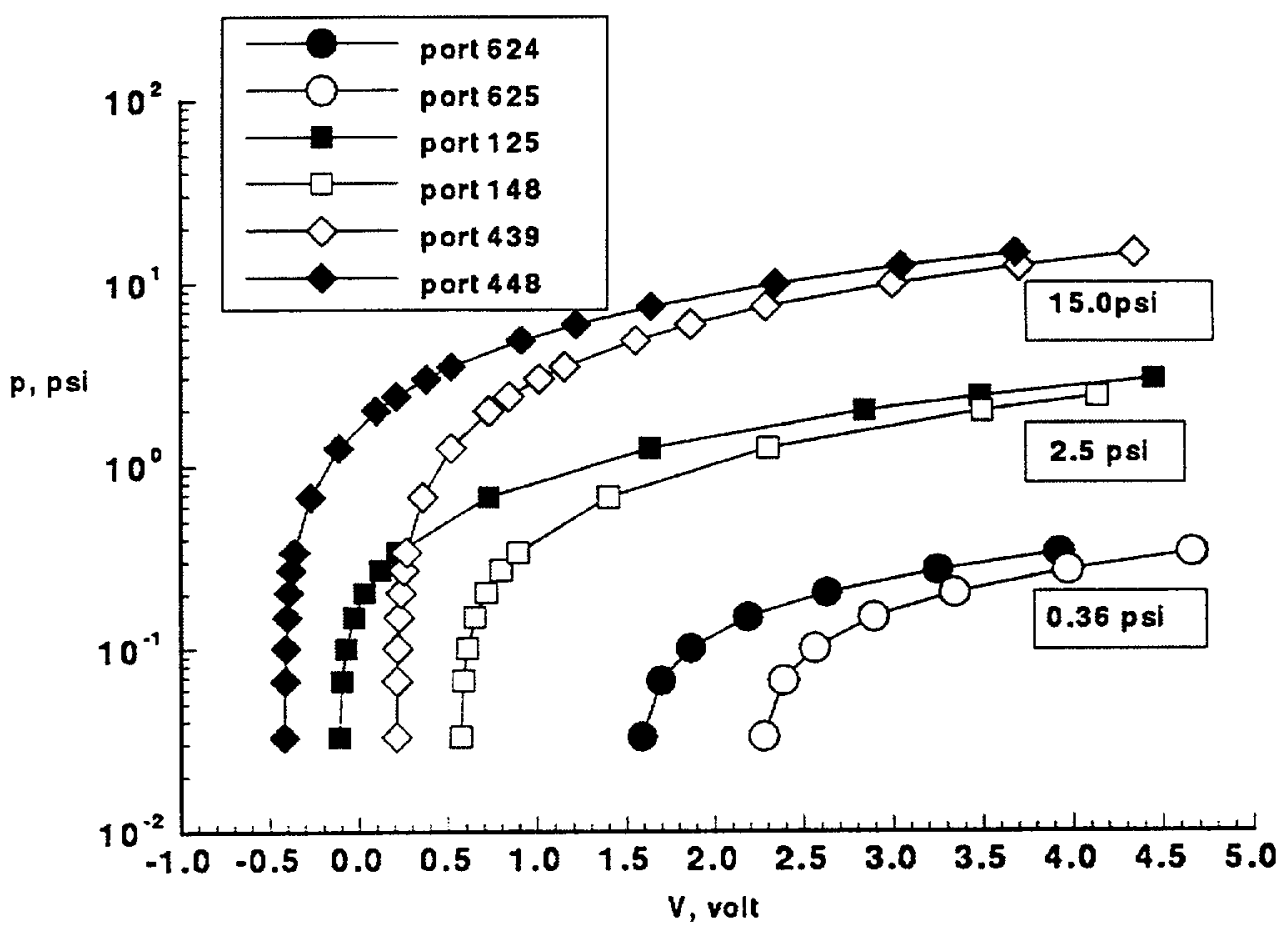

b. Logarithmic scale.

Figure 2.- Typical electronically-scanned pressure system calibration data. 

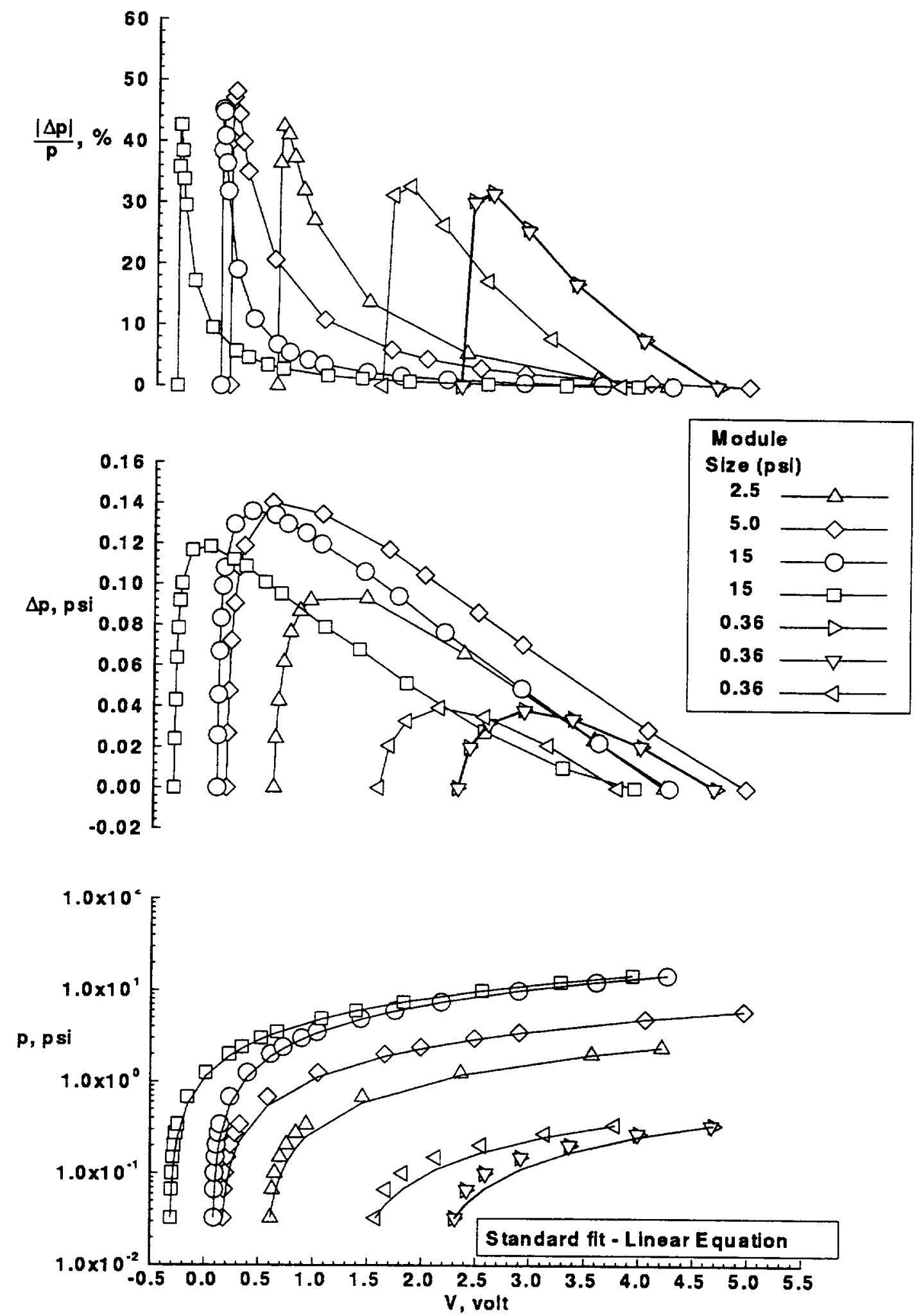

a. Linear fit.

Figure 3.- Standard ESP calibration method using polynomial curve fitting. 


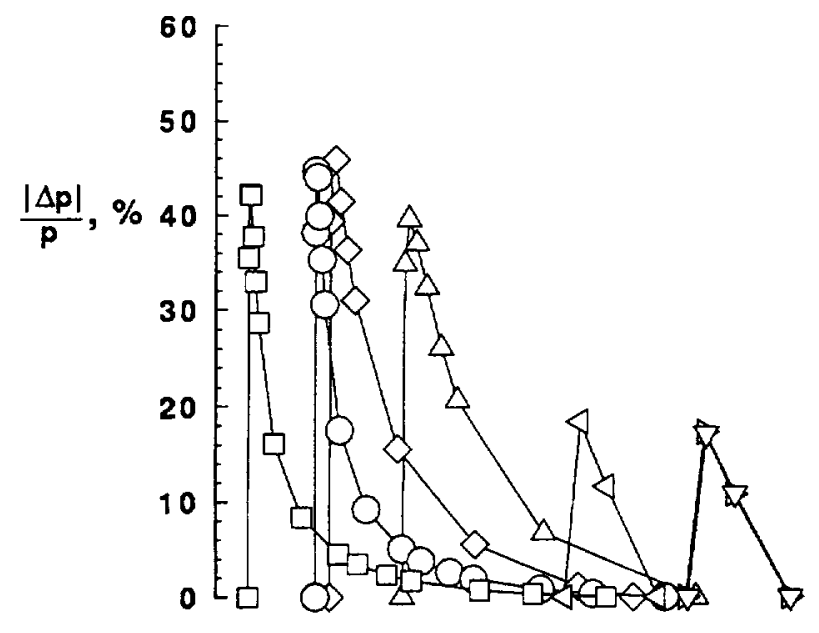

$\square \triangle D \diamond$
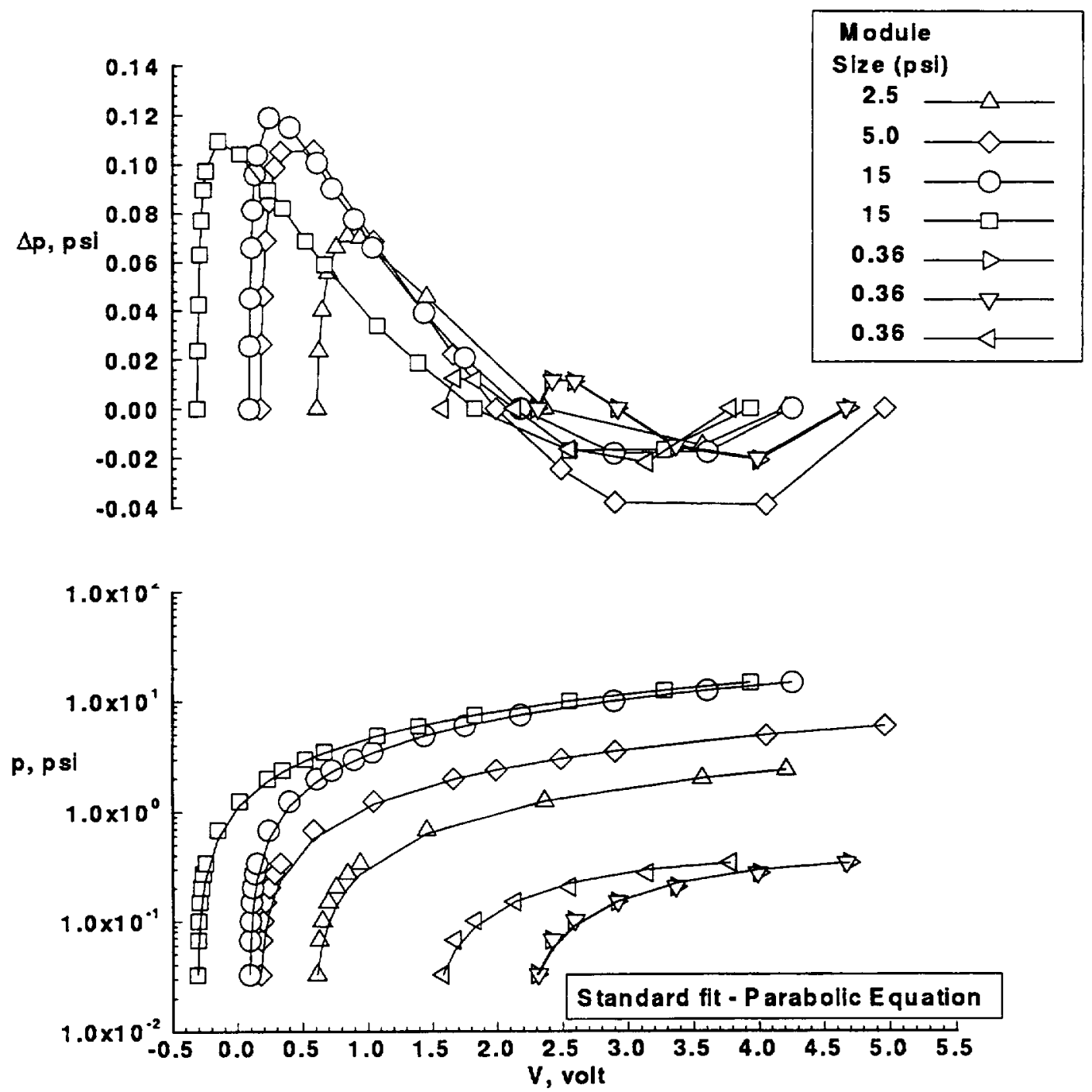

b. Parabolic fit.

Figure 3.- Continued. 

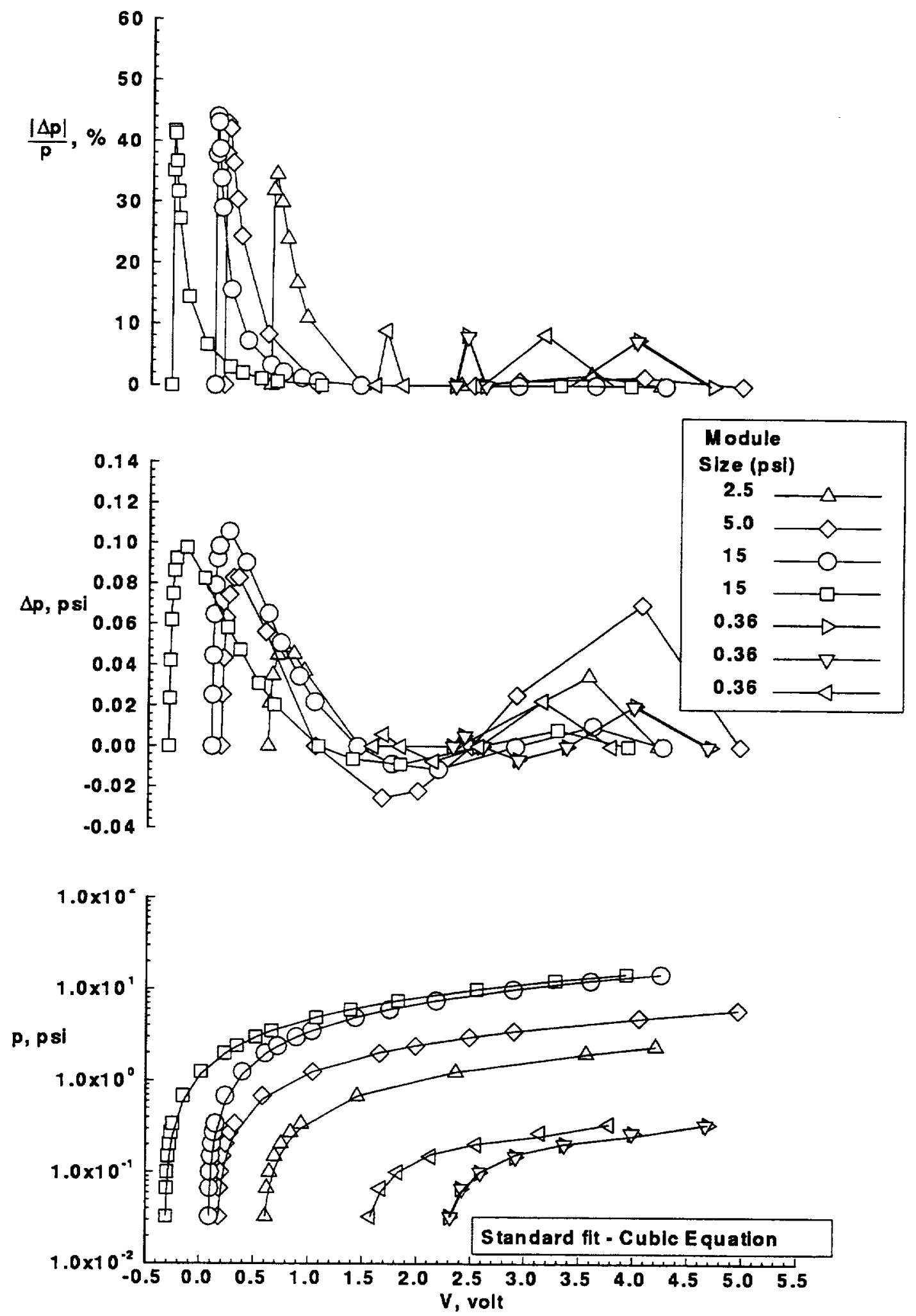

c. Cubic fit.

Figure 3.- Continued. 

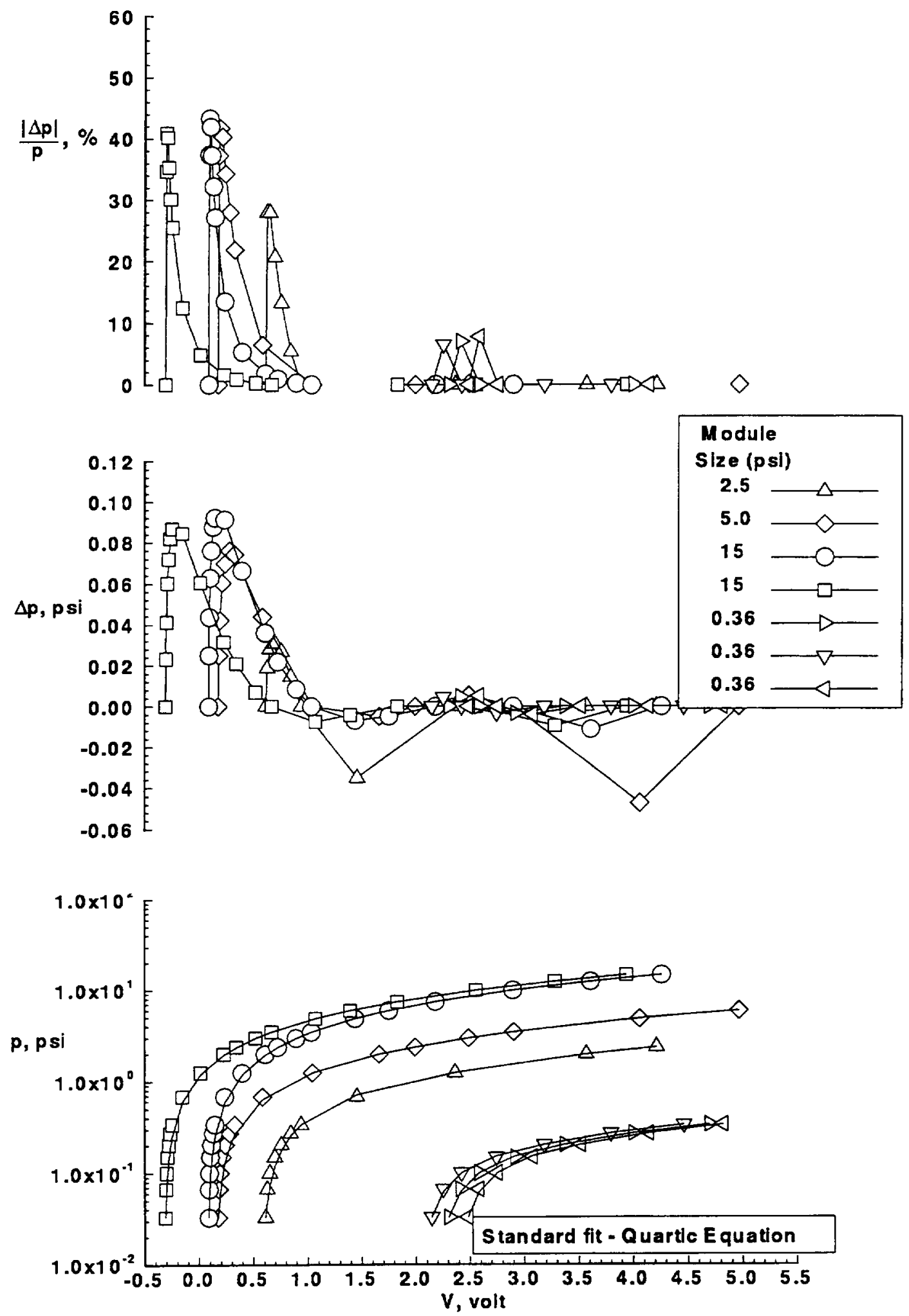

d. Quartic fit.

Figure 3.- Concluded. 

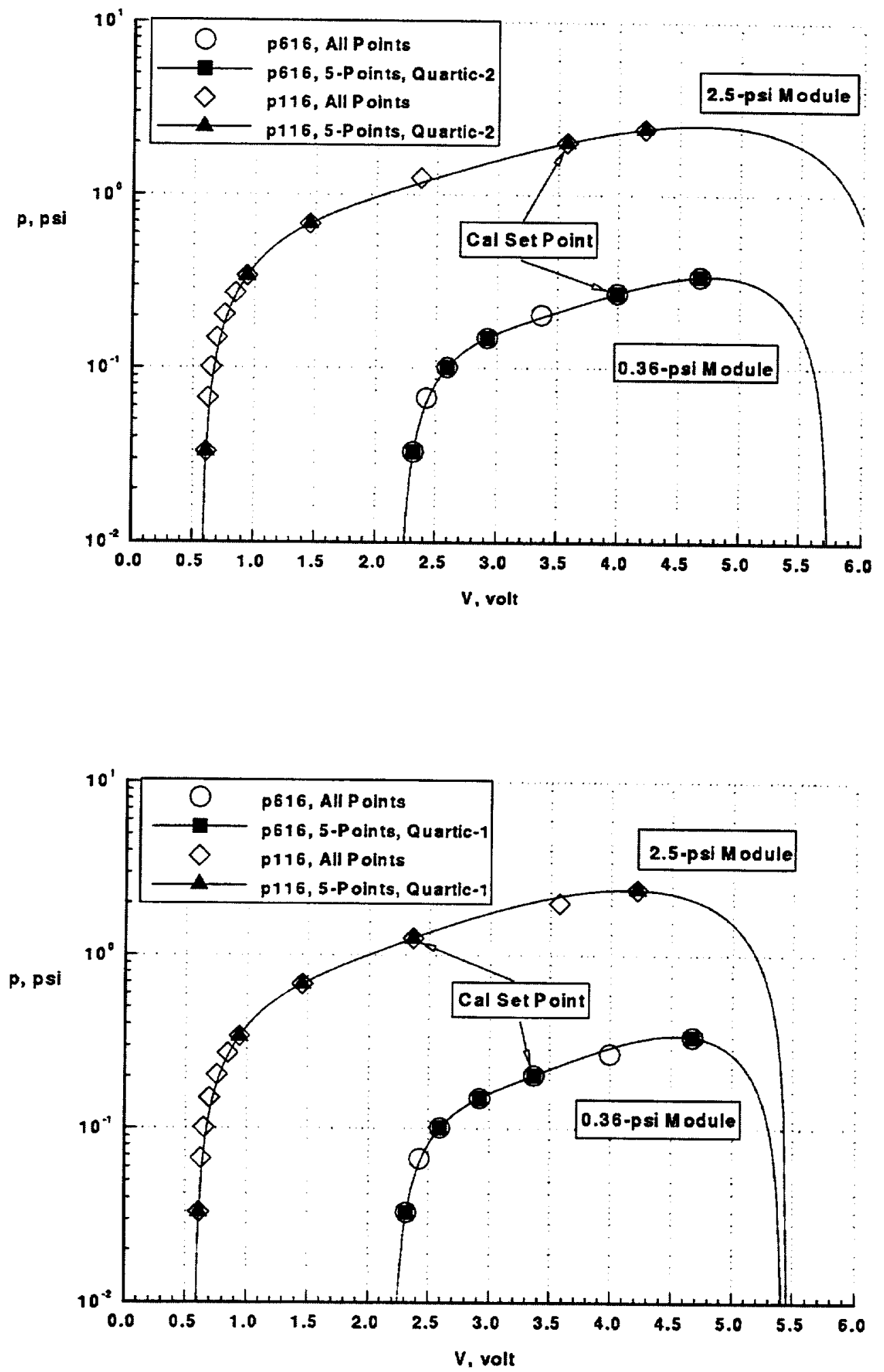

Figure 4.- Effect of calibration pressure location on standard-method ESP calibration curve. 

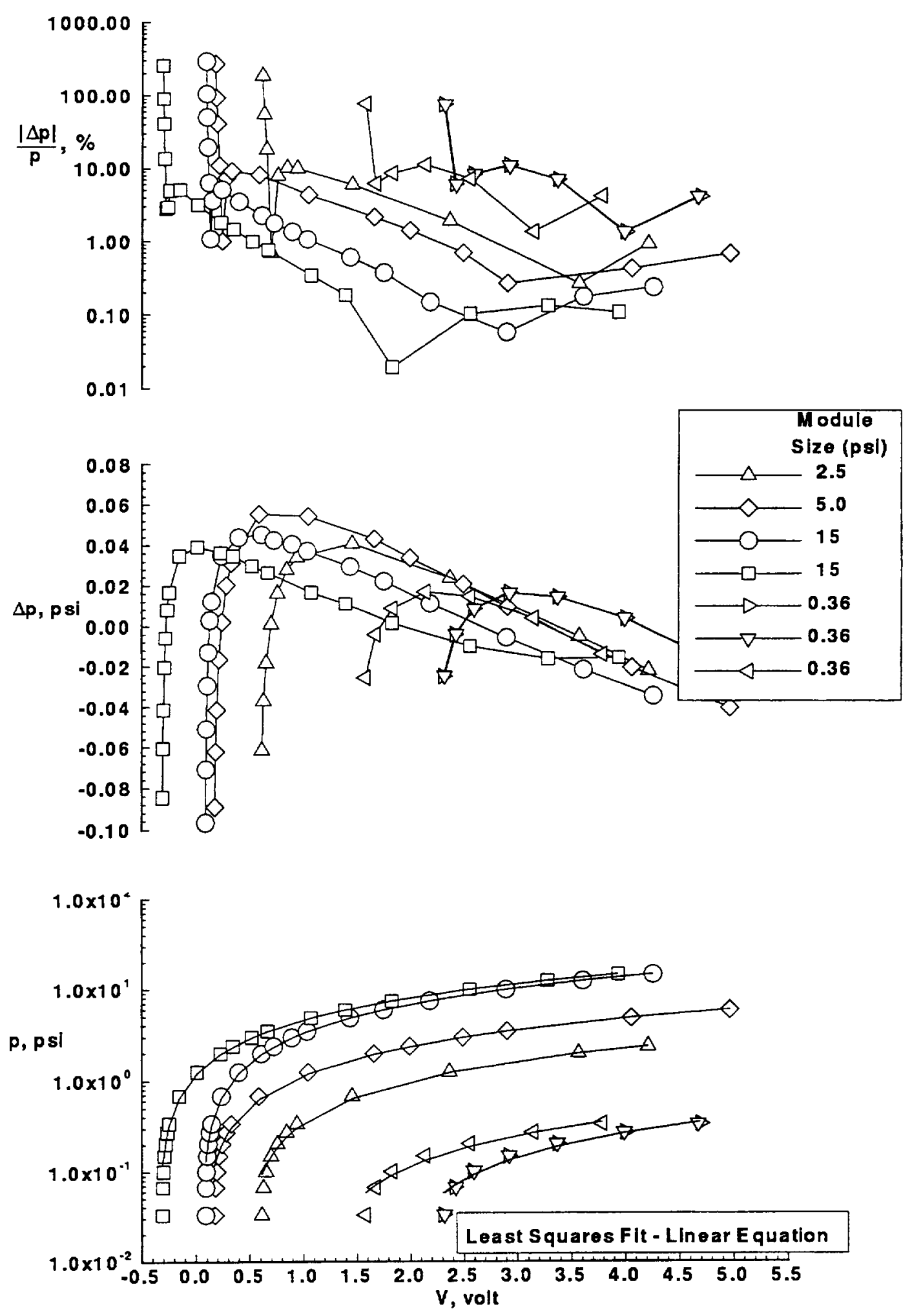

a. Linear fit.

Figure 5.- ESP calibration method using method of least squares fitting. 

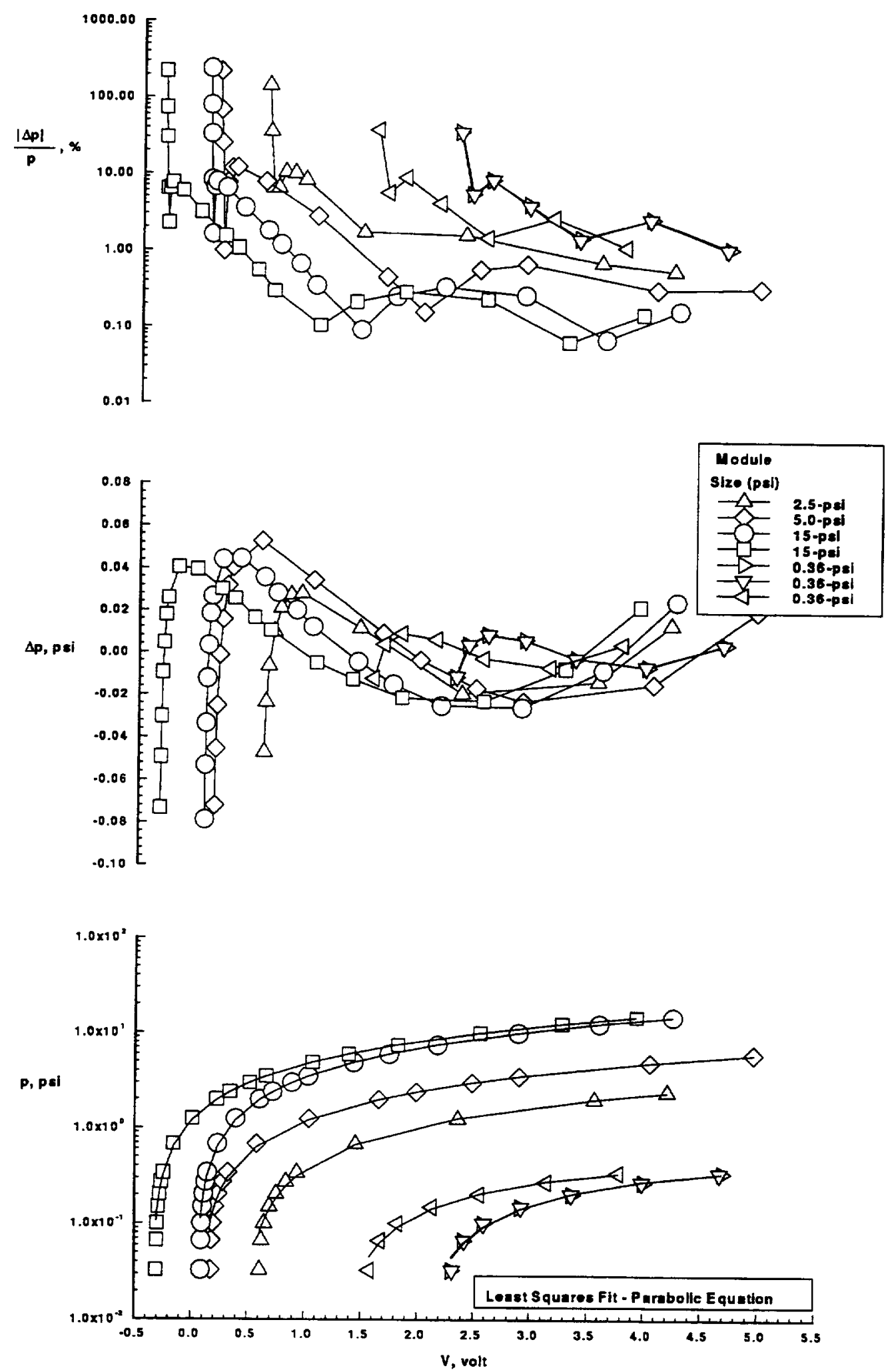

b. Parabolic fit.

Figure 5.- Continued. 

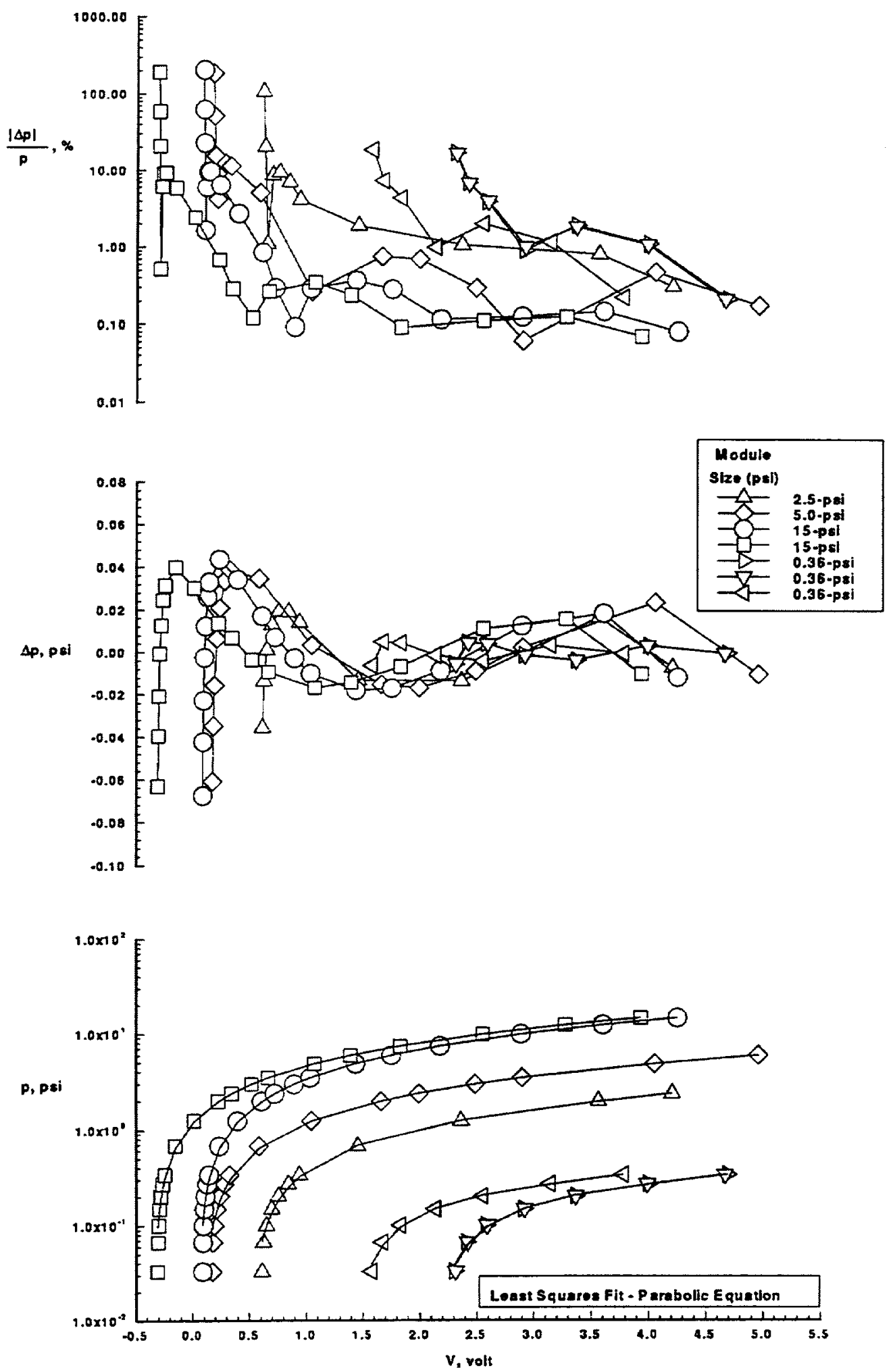

c. Cubic fit.

Figure 5.- Continued. 

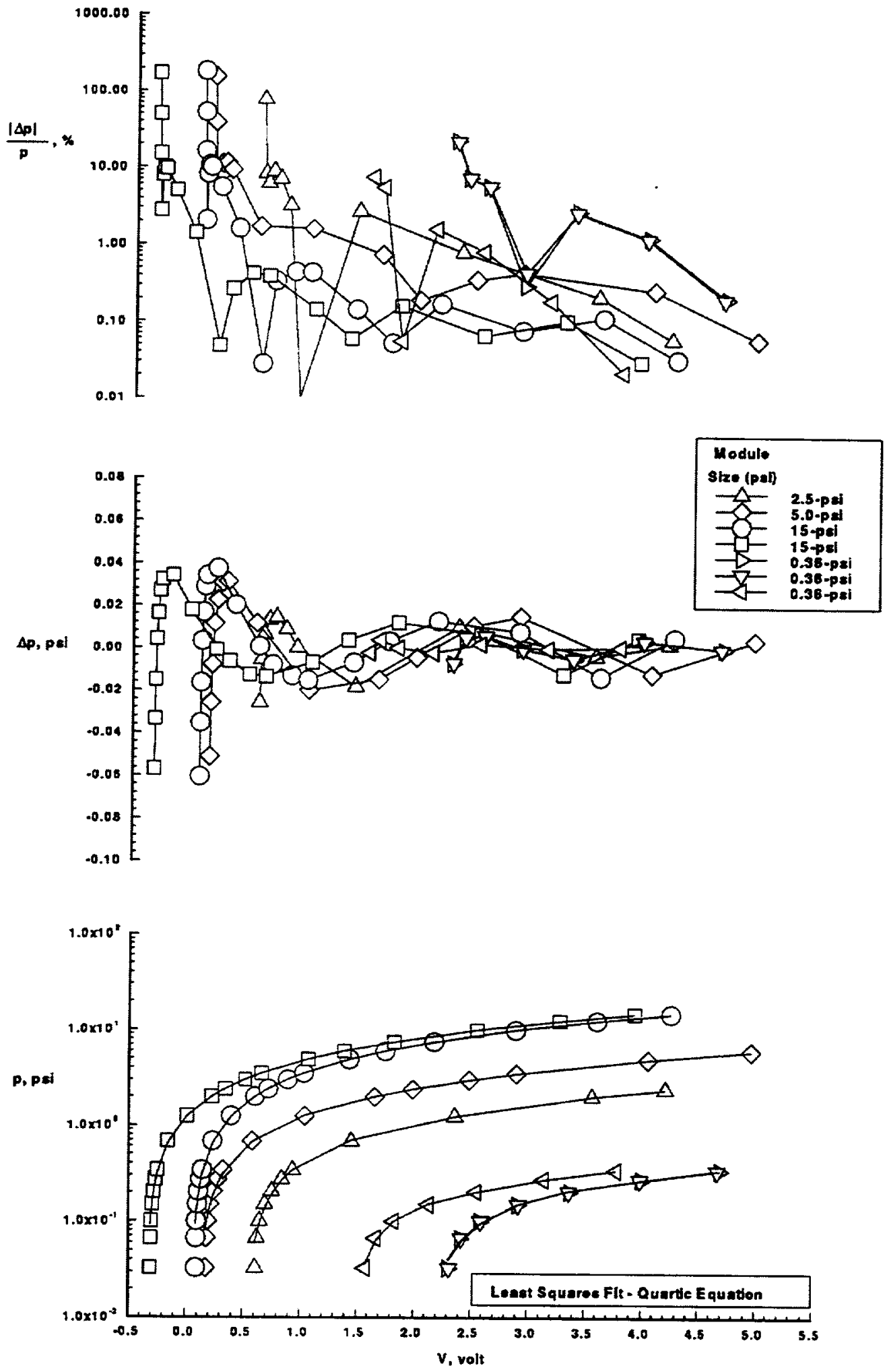

d. Quartic fit.

Figure 5.- Concluded. 

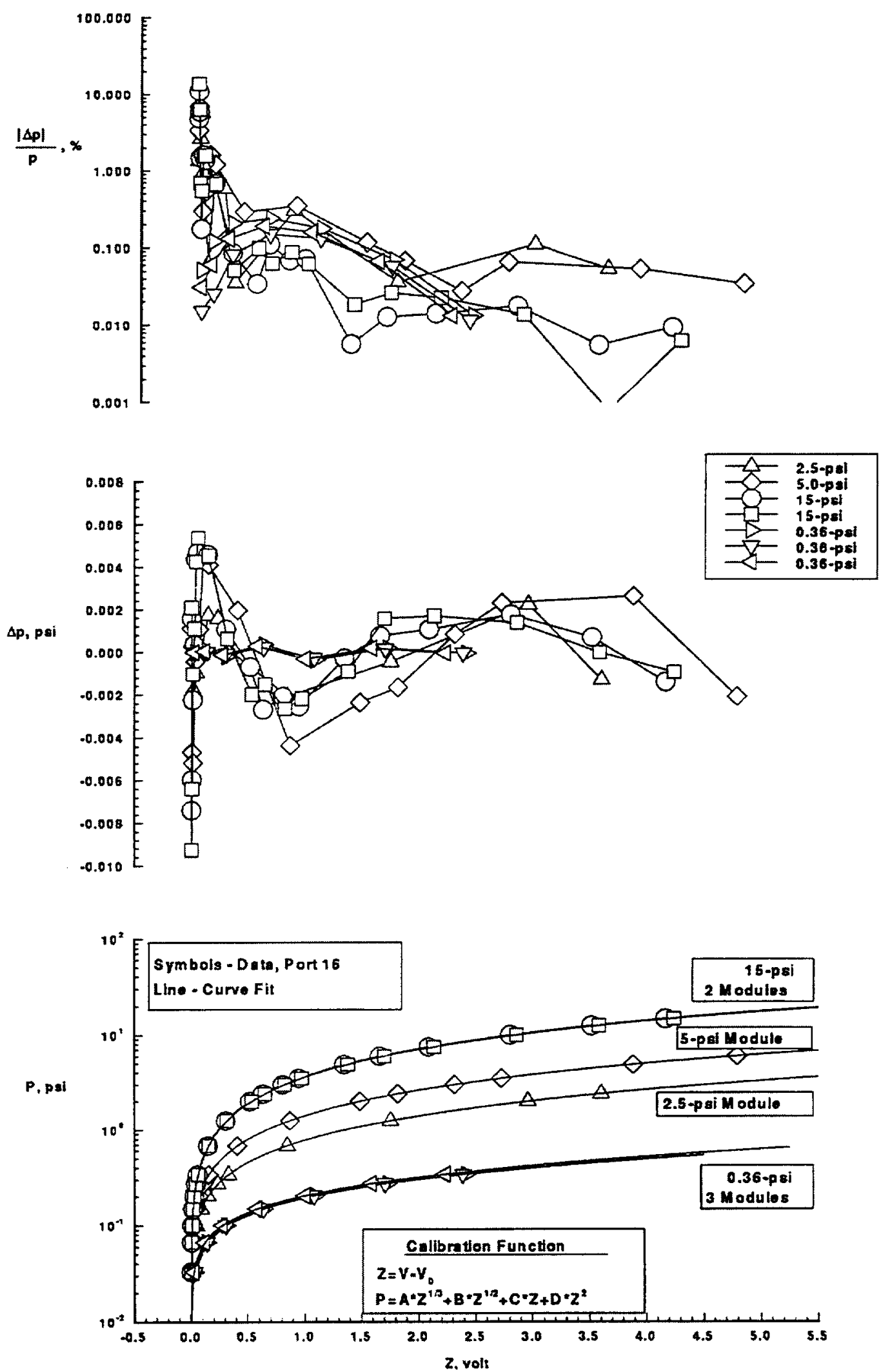

Figure 6.- Improved functional representation of ESP calibration data. 


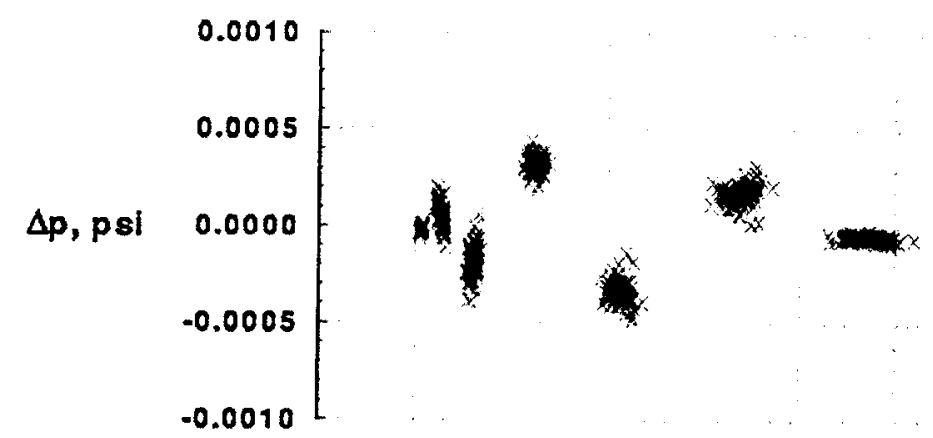

$0.36 \mathrm{psi}$

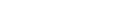

$\Delta p, p s i$
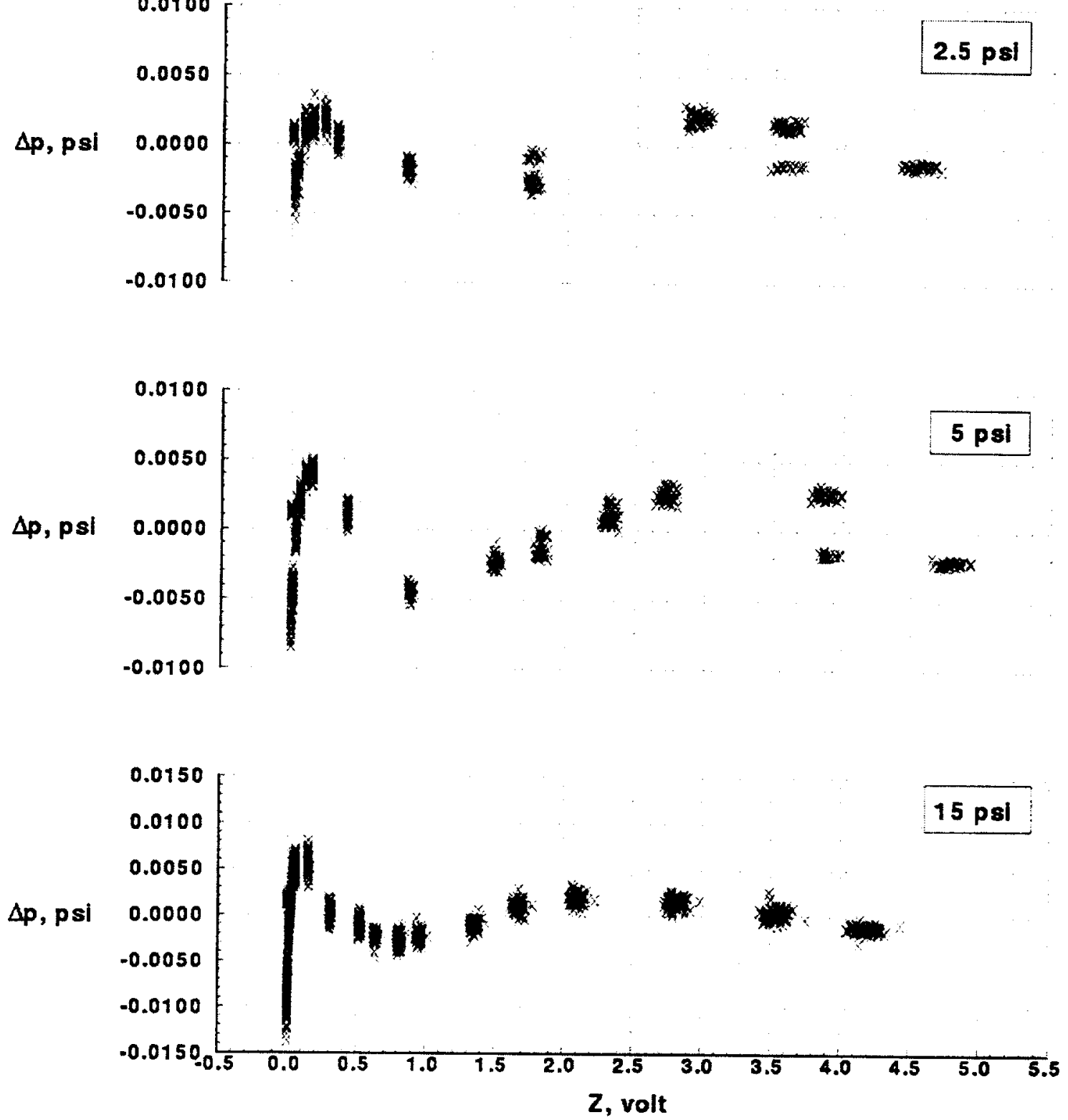

Figure 7.- Improved-method residual error distribution for all module ports. 

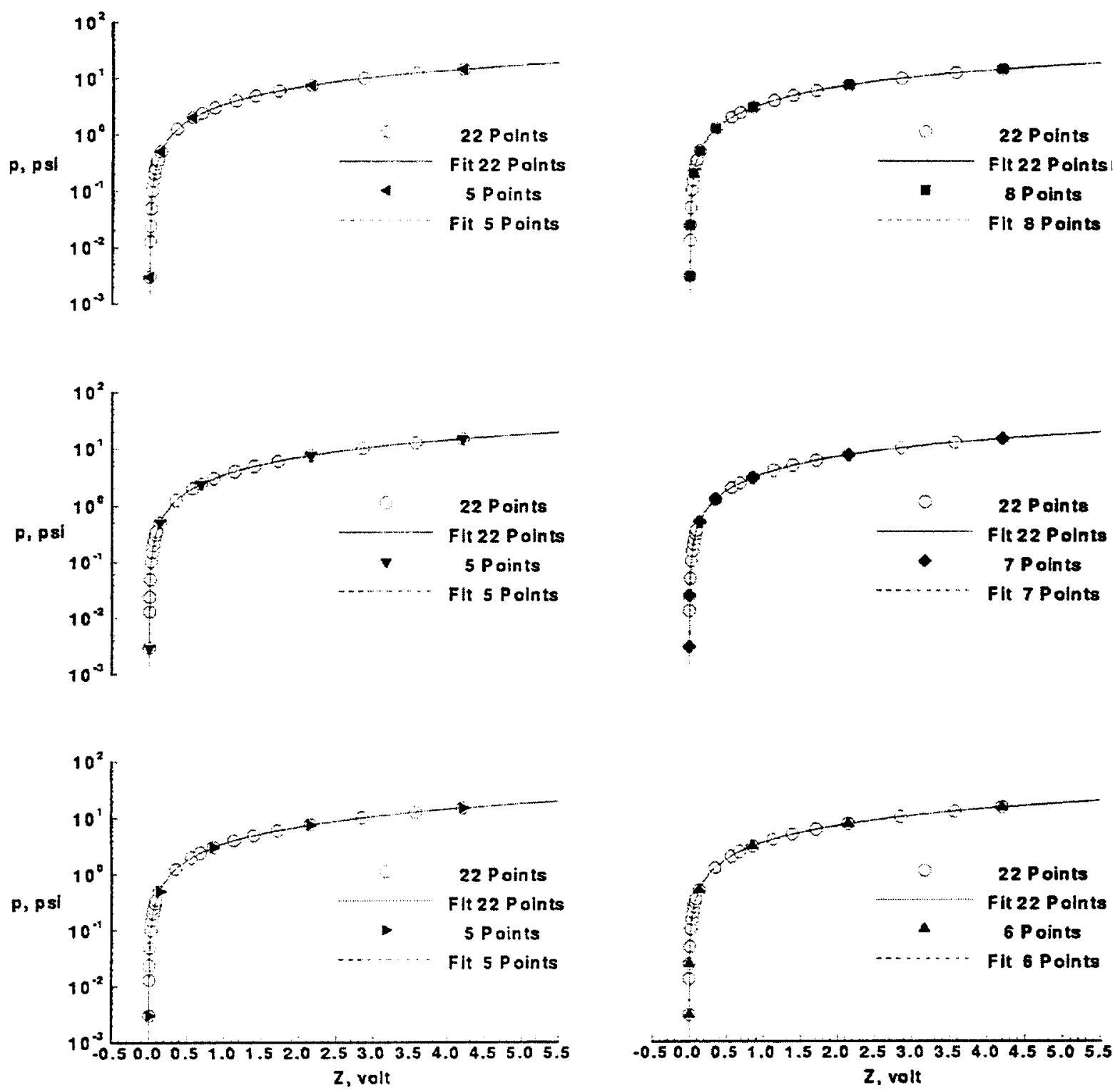

Figure 8.- Effect of number and distribution of calibration points on improvedmethod calibration curves. 


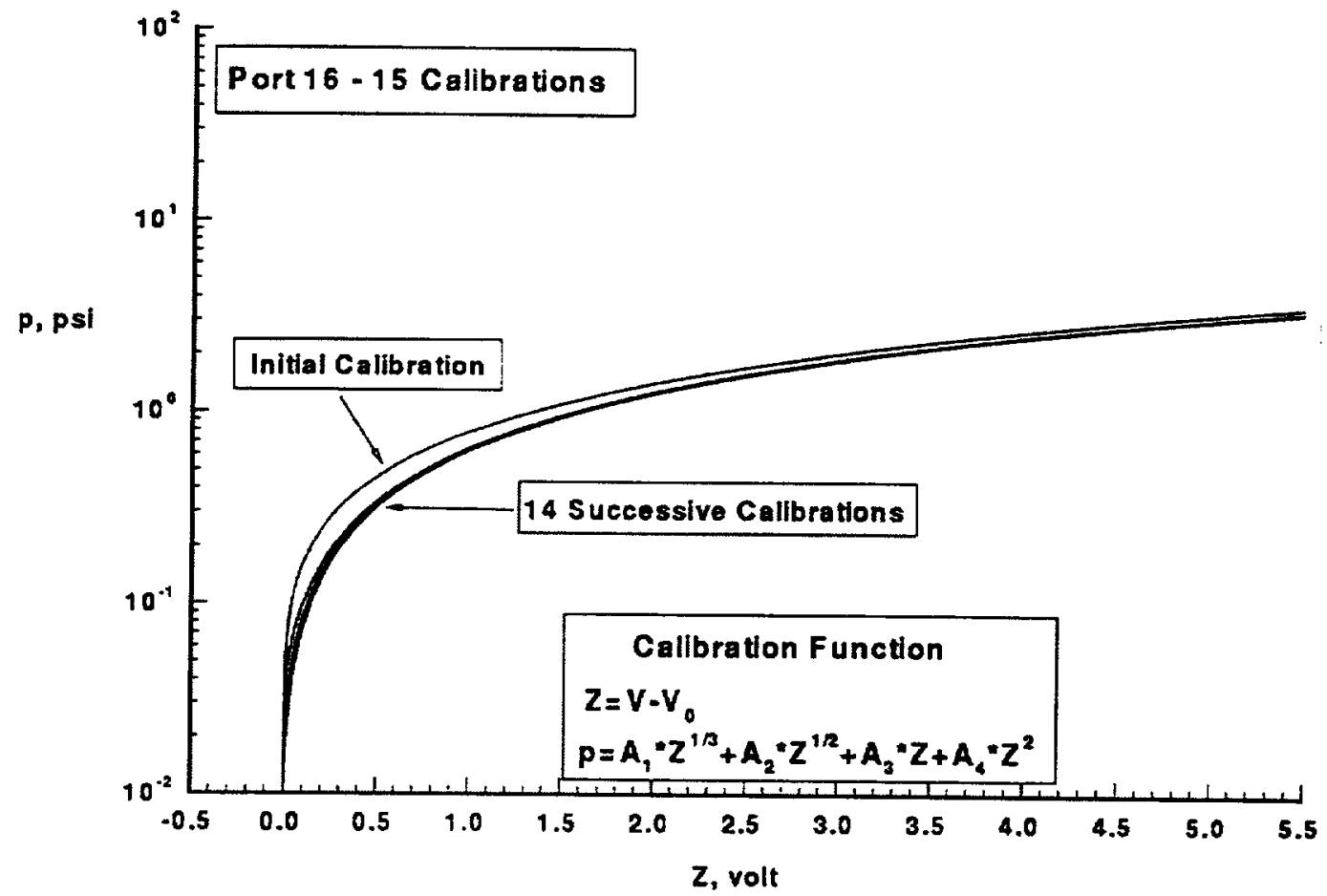

Figure 9.- Comparison of improved-method calibration curves, 2.5-psi module.

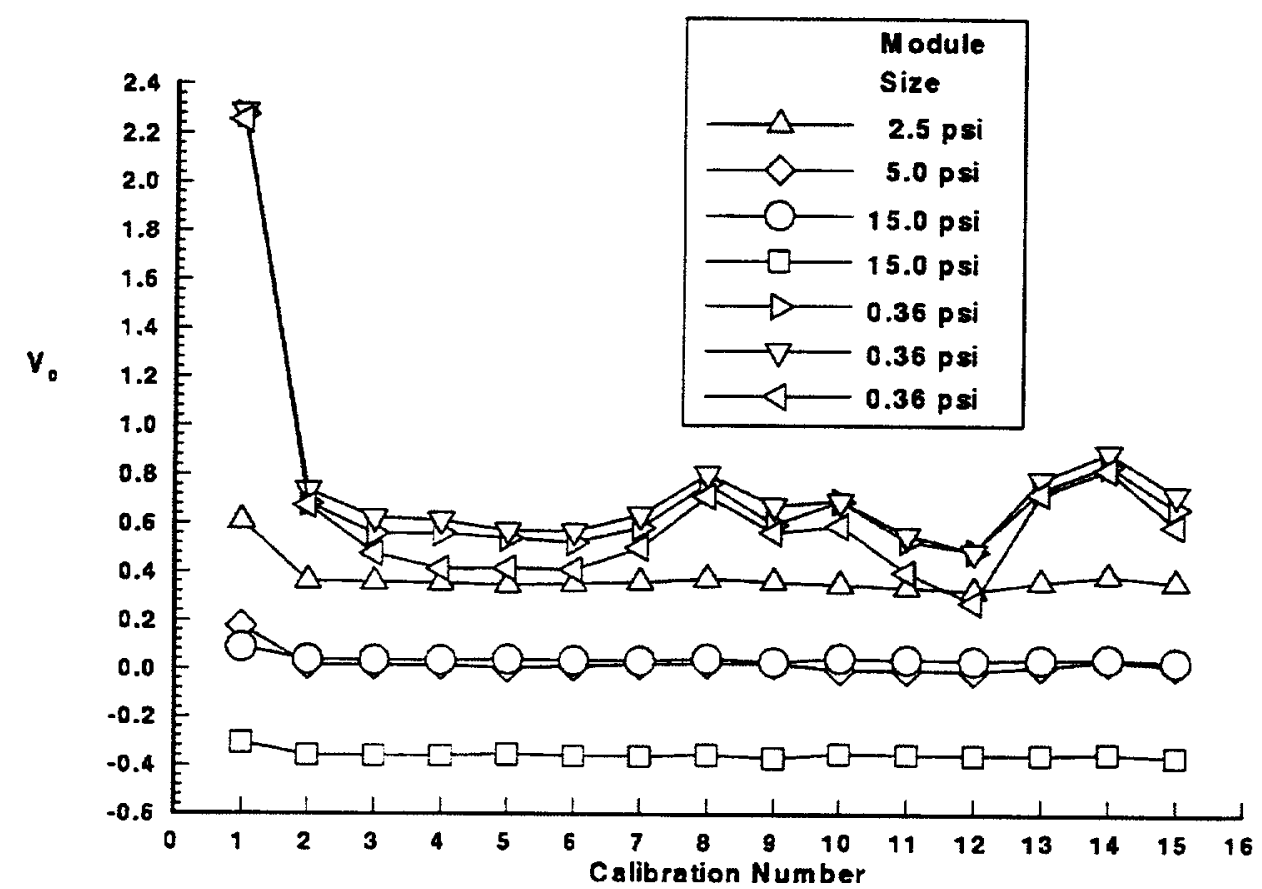

a. Zero-pressure offset voltage, $V_{0}$.

Figure 10.- Historical variation of zero-pressure offset voltage and calibration coefficients. 


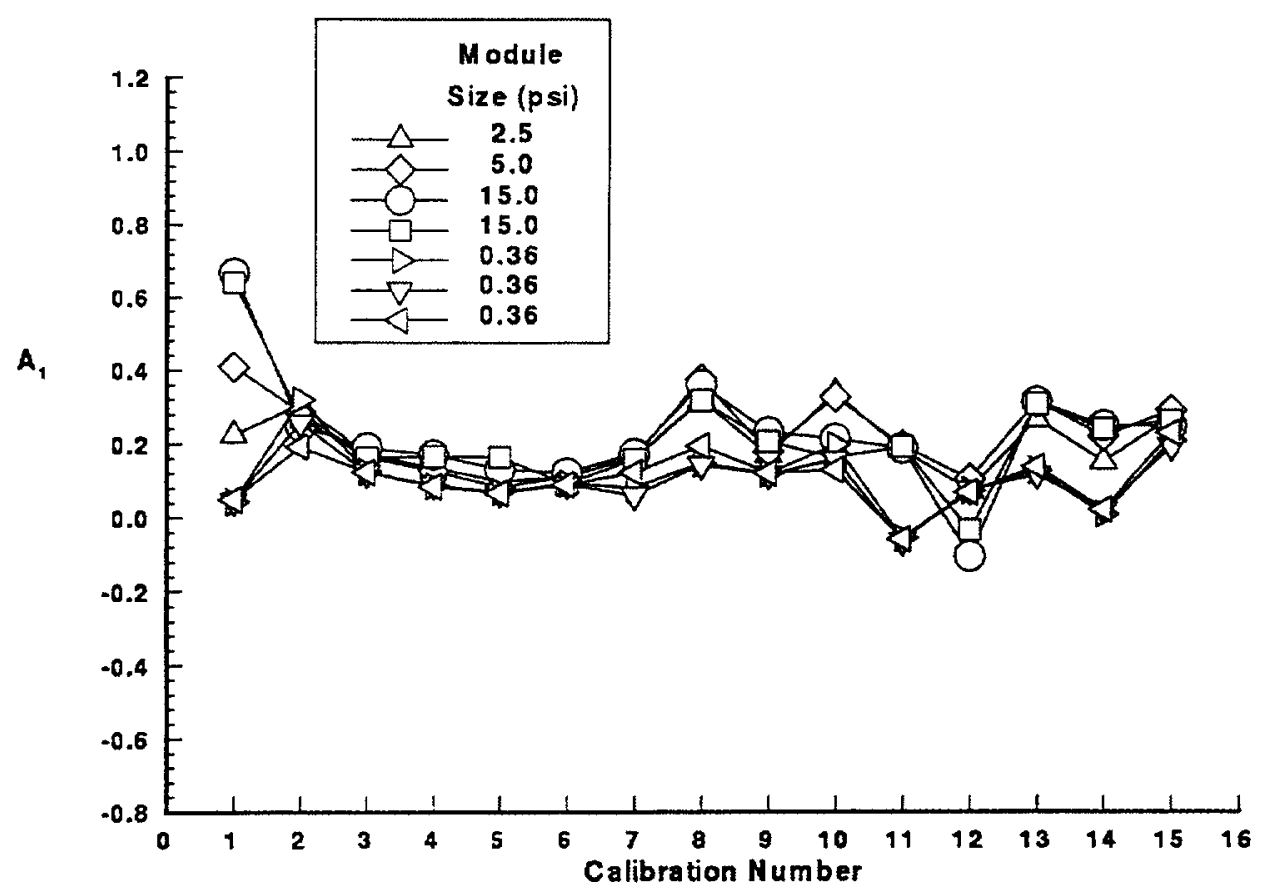

b. Coefficient $A_{1}$.

Figure 10.- Continued.

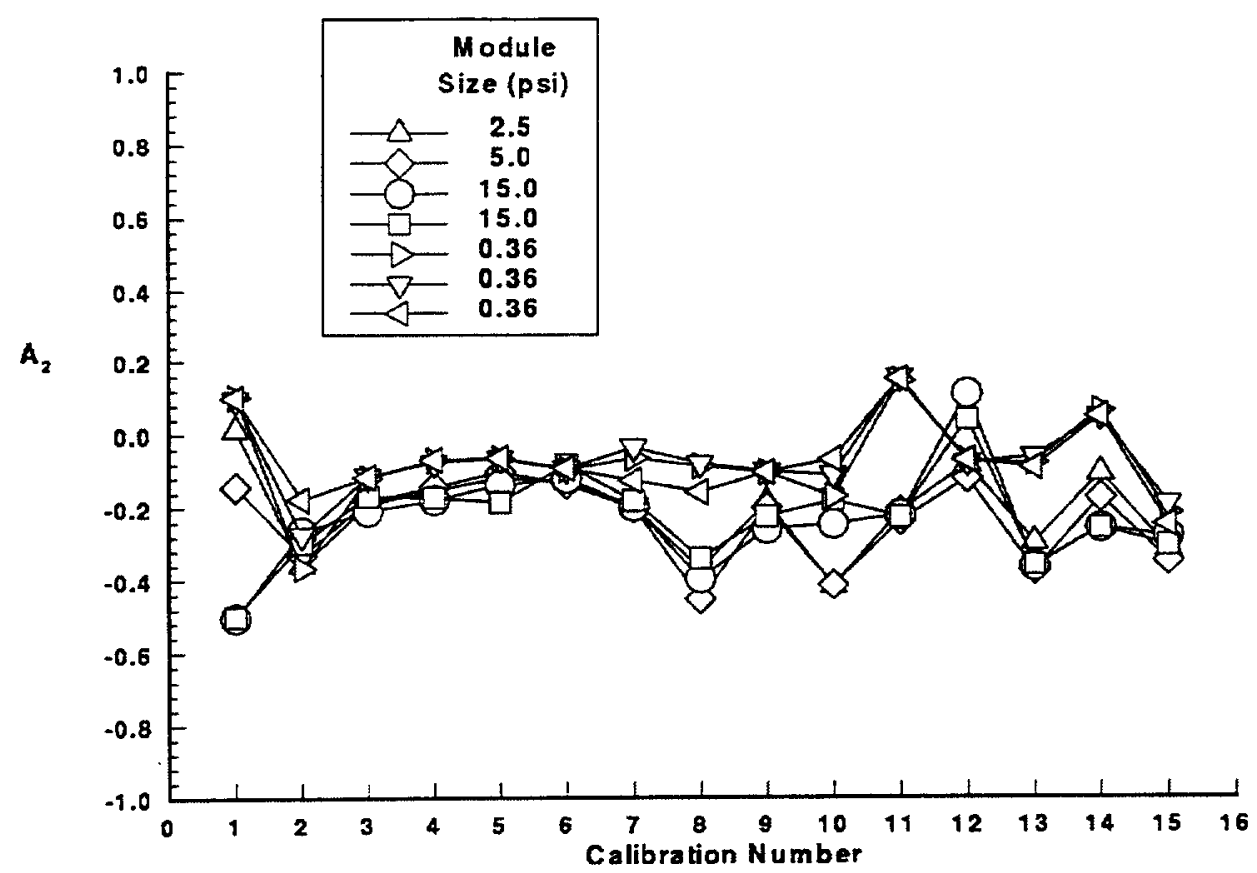

c. Coefficient $A_{2}$.

Figure 10.- Continued. 


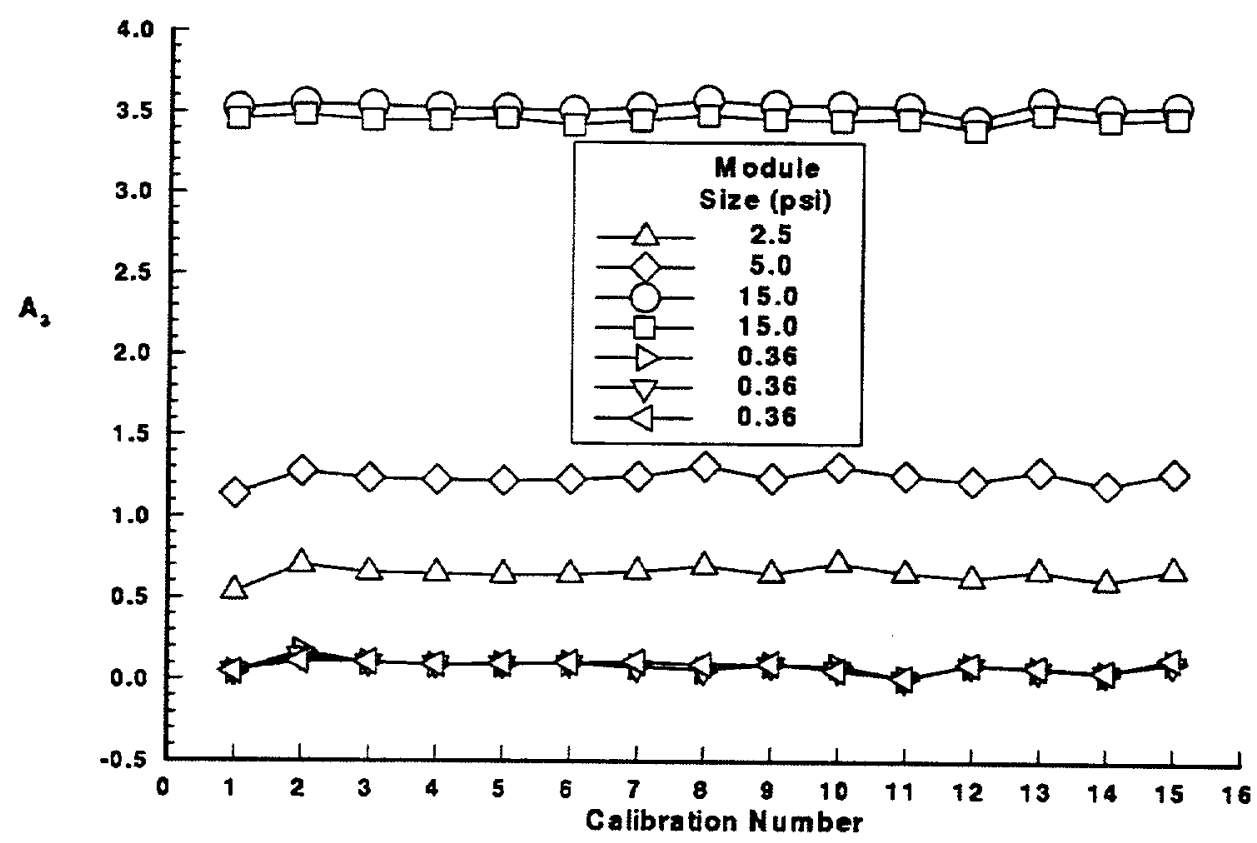

d. Coefficient $A_{3}$.

Figure 10.- Continued.

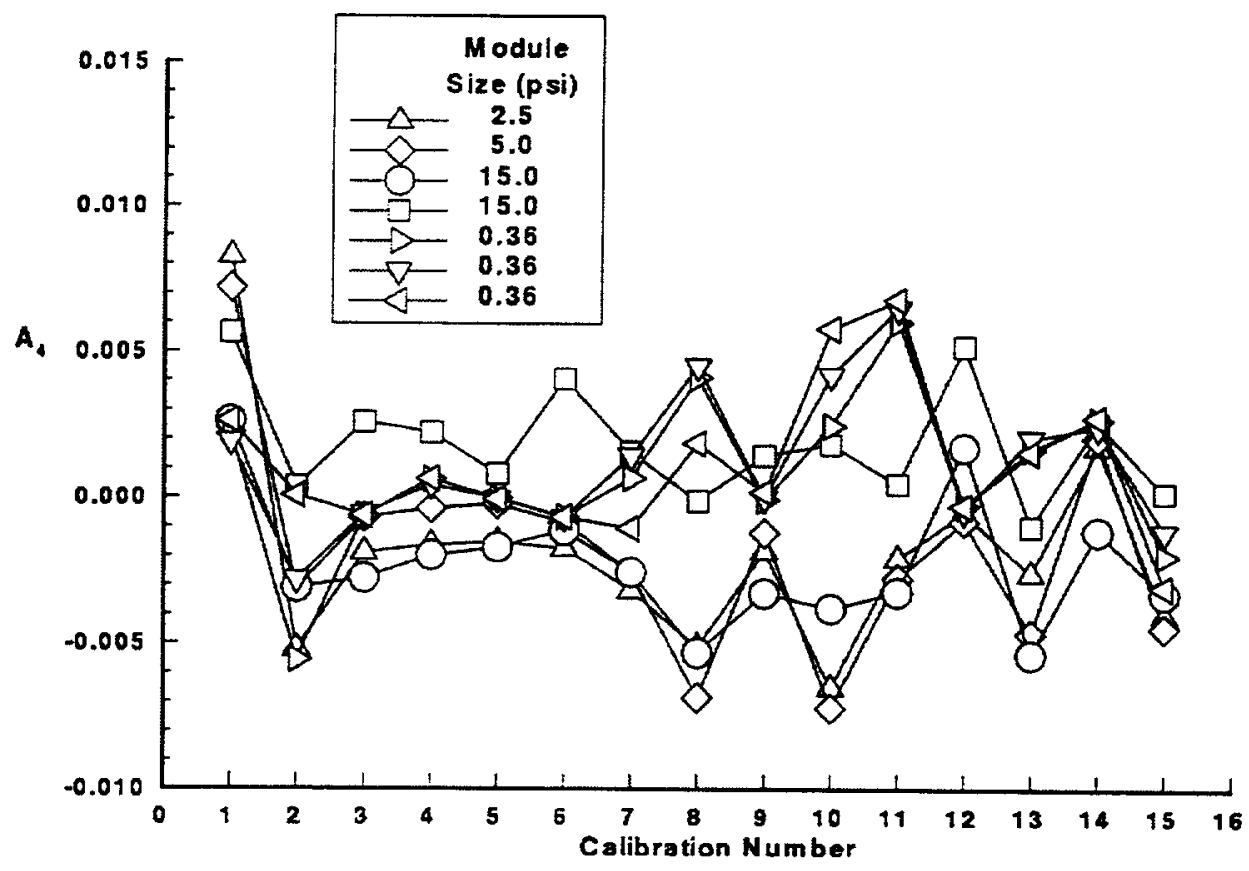

e. Coefficient $A_{4}$.

Figure 10.- Concluded. 


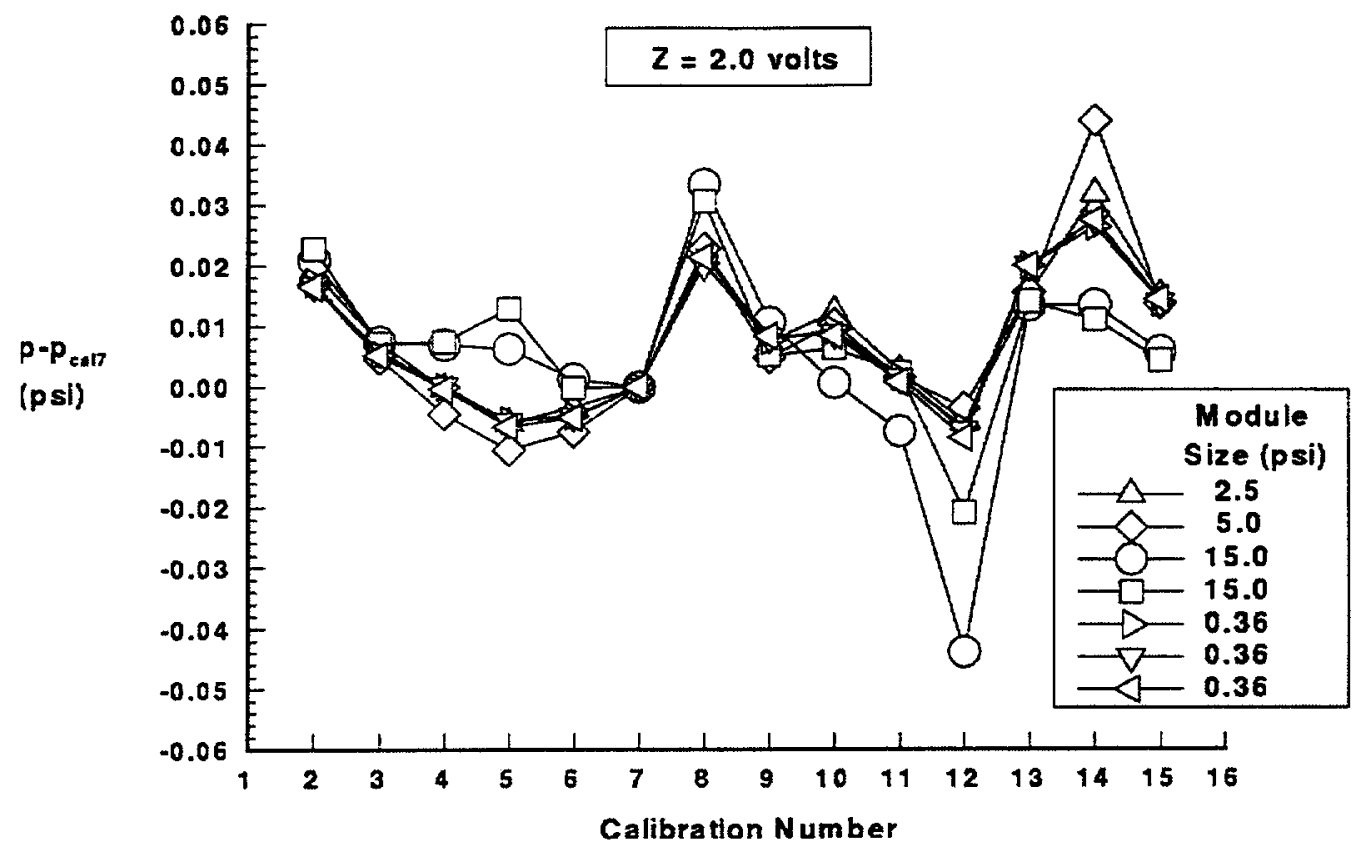

Figure 11.- Historical variation of incremental ESP calibration curves at $Z=2.0$ volts.

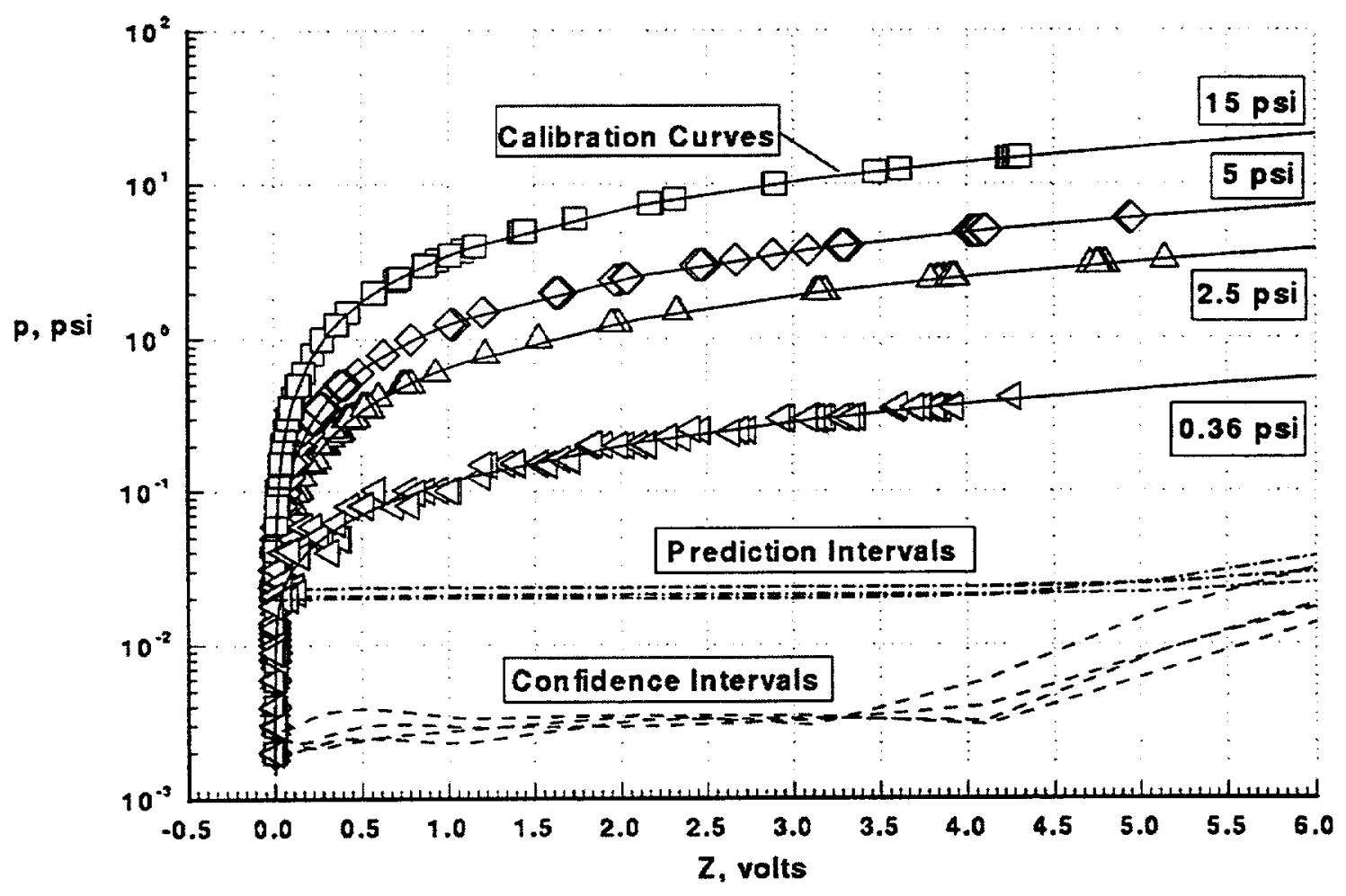

Figure 12.- Confidence and prediction intervals for all pressure ranges. 


